\title{
Multiconvolutional Approach to Treat the Main Probability Distribution Functions Used to Estimate the Measurement Uncertainties of Metallurgical Tests
}

\author{
Ion Pencea
}

Additional information is available at the end of the chapter

http://dx.doi.org/10.5772/50511

\section{Introduction}

The quality of a metallic product is validated by tests results. Thus, the product quality is depicted by the quality of the testing results that furthermore depends on several factors such as: the incomplete knowledge about measurand, the adequacy of the testing method in relation to measurand, the equipment adequacy for method, the human factor; the statistical inference, etc. The influence factors of a measurement process, whether known or unknown, may alter the result of a measurement in an unpredictable way. Thus, a test result bears an intrinsic doubt about its closeness to the conventional true value of the measurand, fact which is commonly perceived as uncertainty about the test result. One of the most important tasks for the experimentalist is to specify an interval about the estimated value of the measurand $(\bar{x}),[\bar{x}-U ; \bar{x}+U]$ in which the true (conventional) value $(\mu)$ could be found with specified confidence level, i.e. the probability $(p)$ that $\mu \in[\bar{x}-\mathrm{U} ; \bar{x}+\mathrm{U}]$. The practice exigency requires $p \geq 95 \%[1,2,3]$. The EN ISO 17025 [1] stipulates that the quality of a numeric test result is quantified by the expanded uncertainty $U(p \%)$, where $p$ is the level of confidence $(p \geq 95 \%)$. An alternative specification of $U$ is its level of significance expressed as 1- $p$. In order to obtain a higher quality of the test result, the experimentalist should perform a set of at least 30 repeated measurements [2,4]. In the field of metallurgy this is quite impossible for technical and economical reasons. Therefore, the quality of the test result should be guaranteed by advanced knowledge about the testing method and by other means such as: equipment etalonation, Certified Reference Materials (CRMs) usage and, last but not least, correct uncertainty estimation based on proper knowledge about the probabilistic behavior of the compound random variable derived from a set of repeated 
measurements (arithmetic mean, standard deviation, etc.). This chapter is intended to provide the knowledge for a better statistical evaluation of the outcomes of the metallurgical tests, based on proper selection of the probability density function mainly for compound variable such as arithmetic sample mean, standard deviation, $t$-variable etc. The reader will find in this chapter the derivations of the Gauss, Student, Fisher-Snedecor distributions and also several compound ones. The derivations are intended to provide the information necessary to select the appropriate specific distribution for a measurand. Furthermore, the chapter addresses the uncertainty estimation based on multiconvolutional approach of the measurand by presenting a case study of Rockwell $C$ hardness test, that reveals the superiority of the statistical inference based on the approach proposed in this chapter.

\section{Probability density functions for experimental data analysis}

\subsection{Elements of Kolmagorov's theory of probability}

According to Kolmogorov's theory [4, 5], the behavior of a random experiment or phenomenon can be mathematically modeled in the frame of class theory using the sample space, the event class and the probability function [5, 6]. Kolmagorov's theory addresses experiments, phenomena or classes of entities having likelihood behavior that can be repeated under the same condition as many times as needed. The testing of the occurrence of an event will be considered generically as being an experiment or probe. The sample space or sample universe $(E)$ is the entire class of outcomes $e_{i}, i=\overline{1, n}$, of an experiment that are mutually exclusive events, respectively

$$
E=\{e i\} i=\overline{1, n} \text { where } e_{i} \cap e_{j}=\phi \forall i \neq j, i, j=\overline{1, n}
$$

An event $A$ is a part of the $E$, i.e. $A \in \mathcal{P}(\mathrm{E})$. The probability of an event is a function defined on $\mathcal{P}(E)$ i.e. $\mathrm{P}: \mathcal{P}(E) \rightarrow[0 ; 1]$, which satisfies the following axioms:

$$
\begin{gathered}
P(A) \geq o ; \forall a \in \mathcal{P}(\Omega) \\
P(E)=1 \\
P(A \cup B)=P(A)+P(B) ; \text { if } A \cap B=\phi
\end{gathered}
$$

\subsection{Discrete and continuous random variables}

A discrete random variable is associated to an experiment that gives finite or countable elementary outcomes, having well defined probabilities. The sum of the probabilities of the discrete sample space must be one. To a finite set of outcomes of an experiment, a discrete random variable $X$ is assigned, which is represented as:

$$
X\left(\begin{array}{lllll}
x_{1} & x_{2} & \ldots \ldots & \ldots & x_{n} \\
p_{1} & p_{2} & \ldots & \ldots & p_{n}
\end{array}\right)
$$

For a countable set of outcomes, a discrete random variable $X$ is expressed as: 


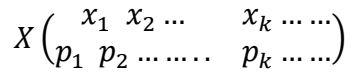

The relationships in (5) and (6) are called the probability distribution functions ( $p d f$ ) of the discrete variable. As it is well known, there are experiments given numeric continuous outcomes. To such experiments, continuous variables are associated. For a continuous random variable $X$, the probability assigned to any specific value is zero, whereas the probability that $X$ takes values in an interval $[a, b]$ is positive. The probability that a continuous random variable $X$ takes values in the $[a, b]$ interval is expressed as $P(a<X<$ $b)$. The probability that a continuous random variable $X$ is less than or equal with a value $x$ is

$$
F_{X}(x)=P(X \leq x)
$$

which is called cumulative distribution function $(c d f)$.

$F_{X}(x)$ should be a continuous and derivable function to fulfill the condition that for any infinitesimal interval $[x, x+d x]$ one can estimate the probability that $X \in[x, x+d x]$ as:

$$
\mathrm{dP}\left((x \leq X<x+d x)=F(x+d x)-F(x)=\frac{F(x+d x)-F(x)}{d x} \cdot d x=\frac{d F}{d x} \cdot d x=p(x) d x\right.
$$

where $p(x)=\frac{d F_{x}(x)}{d x}$ is the density distribution function of $X$. As it is evident, $F_{X}(x)=$ 0 for $x \leq a$ while $F_{X}(x)=1$ for $x \geq b$.

\subsection{Independent and conditional events}

\subsubsection{Conditional probabilities}

Let $E$ be a discrete sample space, containing $n$ elementary events of an experience with probabilistic outcomes. Let be two events $A$ and $B$ of $E$ that contain $k$, respectively $l$ elementary events so that $A \cap B$ contains $m$. Assuming a trial is done and $B$ occurs, then the probability that $A$ occurs simultaneously is the ratio of favorable outcomes for $A$ contained in $B$ and of the possible outcomes of $B$. Thus, the probability of the event $A$, knowing that the compatible event $B$ occurred is named conditional probability of $A$ given $B$ and denoted as $P_{B}(A)$ or $P(A \cap B)$. In the above example the $P_{B}(A)$ is:

$$
P_{B}(A)=\frac{m}{l}=\frac{\frac{m}{n}}{\frac{l}{n}}=\frac{P(A \cap B)}{P(B)}
$$

The probability of $B$ given $A$ is:

$$
P_{A}(B)=\frac{m}{k}=\frac{\left(\frac{m}{n}\right)}{\left(\frac{k}{n}\right)}=\frac{P(A \cap B)}{P(A)}
$$

From Eqs.(9) and (10) it can be derived that

$$
P(A \cap B)=P(A) \cdot P_{A}(A \cap B)=P(B) \cdot P_{B}(A)
$$


Event $A$ is independent of $B$ if the conditional probability of $A$ given $B$ is the same as the unconditional probability of $A, P(A)$ e.g $P_{B}(A)=P(A)$. According to Eg.(11) the probability of two independent event of $E$, let say $A$ and $B$, is:

$$
P(A \cap B)=P(A) \cdot P(B)
$$

\subsubsection{Pairwise and global independence}

If the event $A_{i}, i=\overline{1,3}$ are such that any of pairs are exclusive e.g. $A_{i} \cap A_{j}=\varnothing, \forall i \neq j$ then the events $A_{i}, i=\overline{1,3}$ may be not totally exclusive i.e. $A_{1} \cap A_{2} \cap A_{3} \neq \emptyset$. The classical proof of 3 events pairwise independent but not totally independent was given by S. Berstain $[5,6]$. The events $A_{i} \subset E, i=\overline{1, n}$ are totally independent if for any selection of $k$ events of $E$, written as $\{A s 1, A s 2 \ldots . . . A s k\}$, the following statement is true

$$
P\left(\bigcap_{i=1}^{k} A_{S i}\right)=\prod_{i=1}^{k} P\left(A_{S i}\right)
$$

\subsubsection{Geometric probabilities}

The probability of an event related to the location of a geometric figure placed randomly on a specific planar or spatial domain is called geometric probability. A representative example is that of a disk (D) of radius " $r$ ", that is thrown randomly onto a planar domain $A$ (square of edge length $a$ ) that includes a sub domain $B$ (square of edge length $b$ ) as shown in Figure 1.a. The addressed problem is to estimate the possibility that the disk center falls into the domain $B$. This is the ration between the area of domain $B$ and the area of $A$, i.e. $P(C \in$ $B)=(b / a)^{2}$. If the event consists of $D \cap B$, then the probability is $P(D \cap B)=\left(b^{2}+4 b r+\pi r^{2}\right) / a^{2}$. The examples could be extended to the micro hardness test, e.g. if a field of a steel specimen of area $A$ contains compounds of total area $B$ (Figure 1.b) then the probability that a random indentation the indenter tip impinges on a compound is $p_{B}=B / A$. The most wellknown example of geometric probability is the Buffon's needle problem [7]. Thus, if a needle of length " $2 l$ " is dropped randomly on a horizontal surface ruled with parallel lines at a distance $h>2 l$ apart what is the probability that the needle intersects one of the lines?

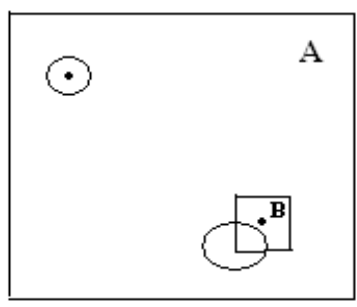

(a)

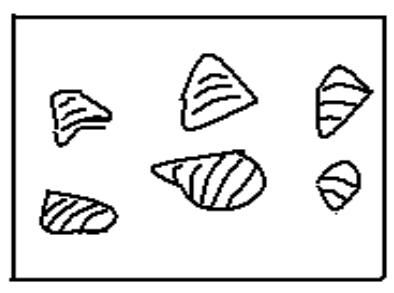

(b)

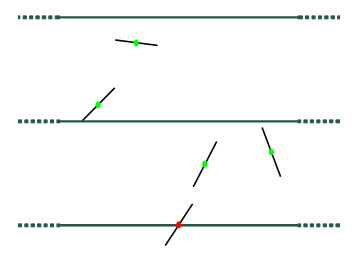

(c)

Figure 1. Schematic representation of the sample space for: a) disk thrown; b) micro-indentation; c) Buffon's needle problem 
The probability that a needle thrown randomly crosses a line is the sum of all favorable probabilities which, in fact, is the integral:

$$
P=\int_{0}^{\pi} \frac{l \sin \theta}{h} \cdot p(\theta) d \theta=\frac{2 l}{\pi h}
$$

The criterion for estimating the correctness of a Buffon's needle experiment is the closeness of $(2 l) /(h P)$ to the value of $\pi$. The geometrical probabilities are used on a large scale in metallography for grain size and grain shape estimation [7]. Their usage may be extended to micro and nano-indentation tests etc.

\subsection{Discrete probability density functions}

Discrete probability deals with events that occur in a countable sample space [1-4]. If the discrete sample contains " $n$ " elementary events $\left\{e_{i} ; i=\overline{1, n}\right\}$ then an "intrinsic" probability value is assigned to each $e_{i}$ while for any event $X$ is attributed a probability $f(x)$ which satisfies the following properties:

$$
\begin{gathered}
f(X) \epsilon[0,1] \text { for all } X \in E \\
\sum_{X \in E} f(x)=1
\end{gathered}
$$

The function $f(e i), i=\overline{1, n}$ that maps an elementar event to the "probability value" in the $[0$, 1] interval is called the probability mass function, abbreviated as $p m f$. The probability theory does not deal with $f\left(e_{i}\right)$ assessing, but builds methods for probability calculation of every event assuming prior known of $f\left(e_{i}\right), i=\overline{1, n}$. The $p m f$ is synonymous with $p d f$ therefore $p d f$ will be used as the abbreviation for the probability density of $X$, which is:

$$
X\left\{\begin{array}{l}
x_{1}, x_{2}, \ldots \ldots \ldots . . . x_{n} \\
p_{1}, p_{2}, \ldots \ldots \ldots p_{n}
\end{array}\right\}
$$

where $\mathrm{x}_{\mathrm{i}}=\mathrm{X}\left(\mathrm{e}_{\mathrm{i}}\right), \mathrm{p}_{\mathrm{i}}=\mathrm{f}\left(\mathrm{e}_{\mathrm{i}}\right) \equiv \mathrm{f}\left(\mathrm{x}_{\mathrm{i}}\right) ; i=\overline{1, n}$

Since the most used pdfs ("pmfs") in the field of metallurgy are Poisson and Bernoulli distribution only these distributions will be addressed in this chapter.

\subsubsection{Poisson scheme}

Let us consider a set of $n$ experiences denoted as: $E=E_{1} x E_{2} x \ldots . x E_{n}$. Each experience has two elementary events, i.e. the variable $X_{i}$ attached to the sample space of $E_{\mathrm{i},} i=\overline{1, n}$ is:

$$
X_{i}\left(\begin{array}{cc}
A_{i} & \overline{A_{l}} \\
p_{i} & q_{i}
\end{array}\right)
$$

where $A_{i}$ is the event of interest of $E_{i}$, having the probability " $p_{i}$ " while $\overline{A l}$ is the contrary one having the probability $q_{i}=1-p_{i}$.

The Poisson's scheme is designed to help estimating the probability of occurrence of $k$ expected outcomes when each experiment $\mathrm{Ei}, i=\overline{1, n}$, of $\mathrm{E}$ is performed once. In this 
instance, assuming that " $k$ " expected events occurred then one can renumber the events starting with these " $k$ " expected and, next, with those (n-k) unexpected as follows:

$$
\left\{A_{j 1}, A_{j 2}, \ldots \ldots \ldots A_{j k}, \bar{A}_{j k+1}, \bar{A}_{j k+2 \ldots \ldots \ldots . .} \bar{A}_{j n}\right\}
$$

The probability of the event $E_{j}=A_{j 1} \cap A_{j 2} \cap \ldots \ldots \cap A_{j+n}$ is the product of the individual $A_{j l}$ events:

$$
P\left(E_{j}\right)=p_{j 1}, p_{j 2}, \ldots q_{j k+1} \ldots . q_{j n}
$$

The event $E_{n}(k)$ is realized for any combination of $k A_{i}, i=\overline{1, n}$, events i.e. for $C_{n}{ }^{k}$ events. Thus to calculate the $P\left(E_{n}(K)\right) \equiv P_{n}(K)$ one must sum all different products consisting of " $k$ " terms of $p_{i}, i=\overline{1, n}$ multiplied by the rest of $q_{i}$ probabilities. This way of calculation is identical with the case of calculating the coefficient of $X^{k}$ of the polynomial:

$$
P_{n}(X)=\left(p_{1} X+q_{1}\right)\left(p_{2} X+q_{2}\right) \ldots\left(p_{n} X+q_{n}\right) \equiv \prod_{i=1}^{n}\left(p_{i} X+q_{i}\right)
$$

This approach of calculations of $P_{n}(k)$ as the coefficient of $X^{k}$ of $P_{n}(x)$ is known as Poisson's scheme.

\subsubsection{Bernoulli or binominal distribution}

The Bernoulli distribution addresses the case of an array of " $n$ " identical experiences, i.e. the particular case of Poisson scheme when $p_{i}=p$ and $q_{i}=q, \mathrm{i}=\overline{1, n}$. In this case, the probability of occurrence of " $k$ " events from " $n$ " trials is:

$$
P_{n}(k)=C_{n}^{k} p^{k} q^{n-k}=\frac{n !}{k !(n-k) !} p^{k} q^{n-k}
$$

where $n !=1 * 2 * 3 * \ldots * n$

The mean value of " $k$ ", denoted as $\mu$, could be calculated as:

$$
M(k)=\sum_{k=0}^{n} k C_{n}^{k} p^{k} q^{n-k}=\sum_{k=1}^{n} n C_{n-1}^{k-1} p^{k} q^{n-k}=\sum_{j=0}^{n-1} n p C_{k-1}^{n-1} p^{j} q^{n-1-j}=n p
$$

The term dispersion is many times used instead of variance.

The variance of the Bernoulli distribution is:

$$
V(k)=\frac{1}{n} \sum_{k=1}^{n}(k-\mu)^{2} \sum \cdot p_{n}(k)=\frac{1}{n}\left(\sum_{k=1}^{n} k^{2} P(k)\right)-\mu^{2}=\mu^{2} p^{2} q^{2}
$$

\subsubsection{Poisson distribution}

Poisson distribution is a particular case of the Bernoulli distribution when the number of events tested is very large, but the probability of the experimental outcome is close to zero i.e. it is the distribution of rare events. In this instance, the mean $\mu=n \cdot p$ is considered a constant quantity that characterizes the distribution as will be shown forwards. According to the Bernoulli distribution the probability of k events realization in a series of $n \rightarrow \infty$ is: 


$$
P_{n}(k)=C_{n}^{k} p^{k} q^{n-k}=\frac{n(n-1) . .(n-k+1}{k !} p^{k}(1-p)^{n-k}=\prod_{i=0}^{k-1}\left(1-\frac{i}{n}\right) *\left(\left[\left(1-\frac{\mu}{n}\right)^{-\frac{n}{\mu}}\right]^{-\mu \frac{(n-k)}{n}}\right.
$$

The limit of $P_{n}(k)$ for $\rightarrow+\infty$, denoted as $P(k)$, is:

$$
P(k)=\lim _{n \rightarrow \infty}\left(P_{n}(k)\right)=\frac{\mu^{k} \cdot e^{-\mu}}{k !}
$$

The dispersion of the Poisson distribution is $D^{2}=\sqrt{\mu}$.

The Poisson distribution is often assigned to the quantum particle counting statistics because the standard deviation (SD) is simply expressed as square root of the mean number $\langle n\rangle$, where $\langle n\rangle$ is the mean of a set of measurements. Many times the SD is estimated as $\operatorname{sqrt}(n)$ using a single test result. But this approach is many times inappropriate to the real situation because the detection probability of a quatum particle is not close to zero.

There are other interesting discrete $p d f s$ for metallurgist as: hypergeometric distribution, negative binomial distribution, multinomial distribution, [1-5] but it is beyond the scope of this chapter.

\subsection{Continuous probability density function}

\subsubsection{Introduction}

The variable $X$ is called a continuous random variable if its values are real numbers as the outcomes of a large class of measurements dealing with continuous measurand such as temperature of a furnace, grain size etc. The sample space for a continuous variable $X$ may be an interval $[a, b]$ on the real number axes or even entire $\mathbb{R}$ space. The probability thar $X$ takes a value in the interval $\left[x_{1}, x_{2}\right]$, denoted as $P\left(x_{1}<X<x_{2}\right)$, is directly proportional with the interval length $\left|x_{2}-x_{1}\right|$ and depends on the intrinsic nature of the experiment to which $X$ was assigned. The local specificity of $X$ is assessed by $P(x<X<x+d x)$, where $d x$ is of infinitesimal length. The probability that $X<x$ is $P(X<x)=F(x)$, which is called cumulative distribution function, abbreviated $c d f$. By definition, the $c d f$ must be derivable because $P(x<X<x+d x)=$ $F(x+d x)-F(x)$ and for any $d x$ the $(F(x+d x)-F(x)) / d x$ should be finite and continuous i.e.

$$
\lim _{d x \rightarrow 0}\left(\frac{F(x+d x)-F(x)}{d x}\right)=p(x)
$$

where $p(x)$ is the probability density function ( $p d f$ ) over the range $a \leq X \leq b$.

The main statistical parameter assigned to a variable $X$ on the base of its $p d f$ is: mean, median, mode, sample variance or dispersion, standard deviation and central moments. The mean of $X$ variable is:

$$
\mu \equiv M(X)=\int_{a}^{b} x \cdot p(x) d x
$$

where $[a, b]$ is the domain of $X$ variable.

The median value of $X\left(x_{m}\right)$ is the value which divides the number of outcomes into two equal parts i.e. $F\left(x_{m}\right)=0.5$. The mode of a $p d f$ is the value $x_{0}$ for which $p\left(x_{0}\right)$ reaches its maximum i.e. $d p\left(x_{0}\right) / d x=0$ 
The variance $V$ of $X$ is expressed as:

$$
V_{X}=\int_{a}^{b}(x-\mu)^{2} p(x) d x
$$

The standard deviation is defined as:

$$
S D_{X}=\operatorname{sqrt}\left(V_{X)}\right.
$$

$S D$ is a measure of the spreading of $X$ values about $\mu$. A set of measurement results are even more centered around $\mu$ as their dispersion is smaller. The central moment of the order $r$ is defined as:

$$
M_{r}(X)=\int_{a}^{b}(x-\mu)^{r} p(x) d x
$$

where $r$ is a natural number and usually $r>2$.

The central moments are used for assessing the skewness and kustoisis of the $p d f s .[1,4]$

\subsubsection{Continuous uniform probability distribution function}

A random continuous variable $X$ has a uniform $p d f$ if it takes values with the same probability in an interval $[a, b]$. The $p d f$ of an uniform variable, abbreviated as updf, is given as:

$$
p(x)=\left\{\begin{array}{c}
\frac{1}{b-a} ; x \in[a, b] \\
0 ; x \notin[a, b]
\end{array}\right.
$$

The $c m d$ of $X$ is:

$$
\int_{a}^{x} f_{x}(t)=F_{X}(x)=\left\{\begin{array}{c}
0 ; x \leq a \\
\frac{x-a}{b-a} ; x \in[a, b] \\
1 ; x \geq b
\end{array}\right.
$$

Usually, a updf is transformed in a standard uniform distribution by the coordinate transformation:

$$
z=\left[x-\frac{(a+b)}{2}\right] \cdot \frac{2}{b-a} \in[-1,1]
$$

which leads to:

$$
f(z)=\left\{\begin{array}{l}
\frac{1}{2} ; x \in[-1,+1] \\
0 ; x \notin[-1,+1]
\end{array}\right.
$$

The $c d f$ of a standard updf is:

$$
f(z)=\left\{\begin{array}{c}
0 ; x<-1 \\
\frac{1}{2}(1+x) ; x \in[-1,+1] \\
1 ; x>1
\end{array}\right.
$$

The graphs of the standard $u p d f, f(x)$, and of its $c d f, F(x)$, are shown in Figure 2. 
The main parameters of a updf are: $\mu=\frac{a+b}{2} ; S D=(b-a) / \sqrt{3} ; \mu_{2 k+1}=0$ while for a standard updf are $\mu=0, S D=\sqrt{3} / 3$. In testing practice, a updf is assigned to an experimental measurand when there is no information or experimental evidences that its values have a clustering tendency. This is the case of a measure device having specified only the tolerance range as for the pyrometer i.e. $\pm 5^{\circ} \mathrm{C}$.

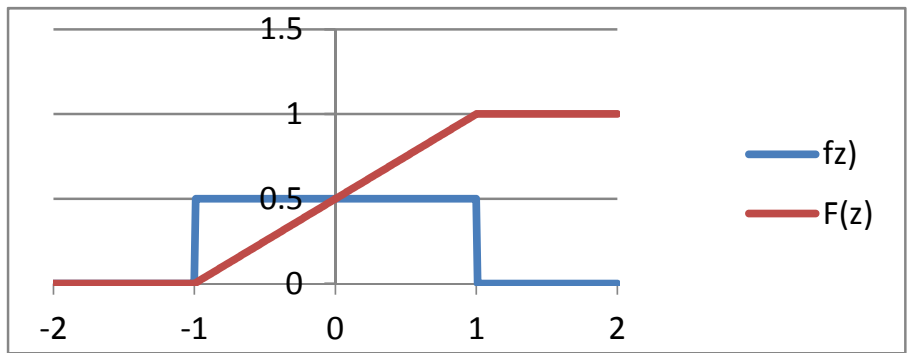

Figure 2. The graphs of the standard $u p d f, f(x)$, and of its $c d f, F(x)$

\subsubsection{Trapezoidal probability distribution function}

A trapezoidal $p d f$ is ascribed to a continuous random measurand if the distribution of its values around the mean is likely to be uniform while at the extremities of the interval the frequency of its occurrence vanishes linearly to zero as is shown in Figure 3.

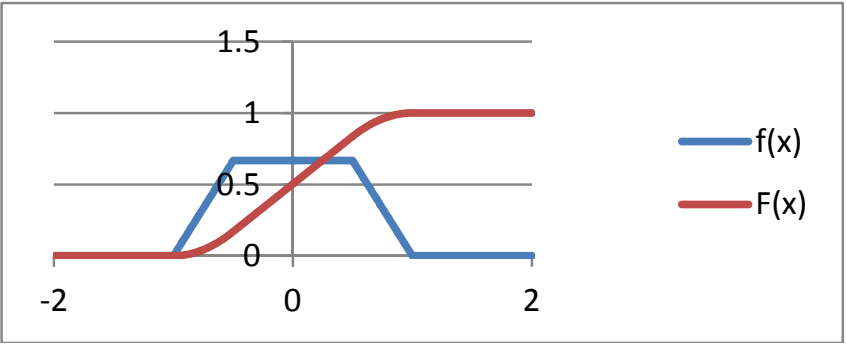

Figure 3. The graphs of trapezoidal symmetric $p d f, f(x)$, and of its $c d f, F(x)$.

The length of the larger base of the trapeze is usually denoted as $2 a$ while the lesser one is $2 b=2 a \beta$. The height of the trapeze $(h)$ is determined by the normalization condition i.e. the area between $f(x)$ and $O x$ axis is 1 . Thus, for a trapezoidal $p d f h=[a(1+\beta)]^{-1}$. In this instance, the isosceles trapezoidal $p d f$ is expressed as:

$$
f(X)\left\{\begin{array}{c}
\frac{x-\mu+a}{a^{2}\left(1-\beta^{2}\right)} ; x \in[\mu-a ; \mu-\beta a] \\
\frac{1}{a(1+\beta)} ; x \in[\mu-\beta a ; \mu+\beta a] \\
\frac{x-\mu-\beta a}{a^{2}\left(1-\beta^{2}\right)} ; x \in[\mu+\beta a ; \mu+a] \\
0 ; \text { otherwise }
\end{array}\right.
$$

The $F(x)$ of a trapeze $p d f$ is obtained by integrating $f(x)$ over the interval $[\mu-a ; x]$. 
The variance of $f(x)$ given by Eq.(37) is:

$$
\mathrm{V}=\mathrm{a}^{2}\left(1+\beta^{2}\right) / 6
$$

The trapezoidal distribution could be considered as the general form for the class of distribution whose graphs are made of linear branches. Thus, for $\beta=1$ the trapezoidal $p d f$ degenerates into a uniform distribution and for $\beta=0$ into a triangular one. Therefore, the triangular distribution will be considered as a particular case of trapezoidal which has the following parameters: $\mu$, and $V=a^{2} / 6$. The triangular distribution is appropriate for the measurand whose values cluster around the mean value. The triangular distribution with the width $2 a$ may be considered as a twofold convolution of uniform distribution of identical length $a$. The same, the trapezoidal distribution can be seen as a convolution of two different uniform distributions. The triangular $p d f$ is mostly used for uncertainty estimation of type B given by an instrument whose tolerance limits are specified.

\subsection{The normal or Gaussian probability distribution function}

Herein will be presented a derivative of normal $p d f$ to emphasize the circumstances of its application The normal or Gaussian $p d f$ was formulated by F. Gauss in 1809 and since then it became the most prominent $p d f$ encountered in testing practice. For example, the result error in a test is usually assumed to follow a normal distribution. The Gaussian function is defined on the entire axis of real number as [4-6]:

$$
f\left(x ; \mu, \sigma^{2}\right)=\frac{1}{\sigma \sqrt{2 \pi}} e^{-\frac{(x-\mu)^{2}}{2 \sigma^{2}}}
$$

where $\mu$ is the mean and $\sigma^{2}$ is the variance of the continuous random variable $\mathrm{X}$.

The Gaussian distribution with $\mu=0$ and $\sigma^{2}=1$ is called the standard normal distribution, denoted by $\Phi(x)$ or $N(0,1)$ which is expressed as:

$$
\phi(x)=\frac{1}{\sqrt{2 \pi}} e^{\frac{-x^{2}}{2}}
$$

$N\left(\mu, \sigma^{2}\right)$ can be expressed as:

$$
f\left(x ; \mu, \sigma^{2}\right)=\frac{1}{\sigma} \Phi\left(\frac{x-\mu}{\sigma}\right)
$$

The function $c d f$ of the standard normal distribution is:

$$
\phi(x)=\frac{1}{\sqrt{2 \pi}} \int_{-\infty}^{x} e^{-\frac{t^{2}}{2}} d t
$$

The integral (40) cannot be expressed in terms of elementary functions but as error function as:

$$
\Phi(x)=\frac{1}{2}\left[1+\operatorname{erf}\left(\frac{x}{\sqrt{2}}\right)\right], x \in R
$$

The $c d f$ of $\mathrm{N}\left(\mu, \sigma^{2}\right), F\left(x ; \mu, \sigma^{2}\right)$, can be expressed as: 


$$
\mathrm{F}\left(\mathrm{x} ; \mu, \sigma^{2}\right)=\Phi\left(\frac{\mathrm{x}-\mu}{\sigma}\right)
$$

The normally distributed variable has a symmetric distribution about $\mu$, which is in the same time the median and the mode. The probability that $X \sim N\left(\mu, \sigma^{2}\right)$ takes values far from $\mu$ (i.e. more than a few standard deviations) drops off extremely rapidly.

From the perspective of the test result analysis, it is important to derive the $f\left(x ; \mu, \sigma^{2}\right)$. An easy and meaningful approach to derive $f\left(x ; \mu, \sigma^{2}\right)$ is based on binomial $p d f$ with $p$ not close to zero and for a very large $n$. In such a case, $P_{n}(k)$ has a maximum for $\tilde{k}=n p$ and drops off very rapidly as $k$ departs from $\tilde{k}$. When $n$ is very large, $P_{n}(k)$ varies smoothly with $k$ and practically $k$ can be considered a continuous variable. Because $P_{n}(k)$ takes significant values only in the vicinity of $k$, then its values will be well approximated by formally constructed Taylor series for $\ln \left(P_{n}(k)\right)$, around $\tilde{k}$ as follows:

$$
\ln \left[P_{n}(k)\right] \cong \ln \left[P_{n}(\tilde{k})\right]+\frac{(k-\tilde{k})}{1 !} \frac{d\left(\ln \left(P_{n}(k)\right)\right.}{d k}\left|\tilde{k}+\frac{(k-\tilde{k})}{2 !} \frac{d^{2} \ln P_{n}(k)}{d k^{2}}\right| \tilde{k}+\frac{(k-\tilde{k})}{3 !} \frac{d^{3} \ln P_{n}(k)}{d k^{3}} \mid \tilde{k}+\cdots
$$

The second term of the right side of the equation is null because $\frac{d P_{n}(\tilde{k})}{d k}=0$.

For deriving the $\ln \left(P_{n}(k)\right)$ it is assumed that the Stirling's first approximation is valid i.e.

$$
\ln \frac{n !}{k !(n-k)} \cong n \cdot \ln (n)--k \ln (k)-(n-k) \ln (n-k)
$$

Based on Eq.(46) the derivative of $\ln P_{n}(k)$ of the $r$ order, $r \geq 2$, was deduced as:

$$
\frac{d^{r} \ln \left(P_{n}(k)\right)}{d k^{r}}=(-1)^{r-1} \cdot \frac{(r-2) !}{k^{r-1}}+\frac{(r-2) !}{(n-k)^{r-1}}
$$

Thus, the terms containing derivative of the order $r>2$ could be neglected in the Taylor's expression of $P_{n}(k)$. With these considerations and taking into account that $\frac{d^{2} \ln P_{n}(\tilde{k})}{d k^{2}}=-\frac{1}{n p q}$ then Eq.(45) can be written as:

$$
P_{n}(k) \cong P_{n}(\tilde{k}) e^{-\frac{(k-n p)^{2}}{2 n p q}}=P_{n}(\tilde{k}) e^{-\frac{(k-\mu)^{2}}{2 \sigma^{2}}}
$$

where $\mu$ is the mean and $\sigma^{2}$ is the variance of binomial $p d f$

The next assumption based on $n$ sufficient larger is to consider $k$ as a continuous variable $X$.

The $X$ variable may be extended on $R$ based on exponential decreasing of the probability that $X$ takes values far from $\mu$. In this instance, Eq.(48) can be written as:

$$
f\left(x ; \mu, \sigma^{2}\right)=C \cdot e^{-\frac{(x-\mu)^{2}}{2 \sigma^{2}}}=\frac{1}{\sigma \sqrt{2 \pi}} e^{-\frac{(x-\mu)^{2}}{2}}
$$

where $\mathrm{C}$ is a constant determined from the normalized condition.

Deduction mathematical expression of the Gauss distribution, $f\left(x ; \mu, \sigma^{2}\right)$, on the base of the binomial $p d f$ clearly shows that the Gaussian $p d f$ is valid for a very large number of experiments. Therefore, by analogy, it is a matter of evidence that Gaussian $p d f$ addresses experiments having a large number of influence factors that give rise to random, unbiased 
errors. Usually, an uncertainty budget comprises a number of influence factors less than 20 . Thus, at the first glance, it results that a normal $p d f$ is not sufficiently justified to be applied in such a case. On the other hand, if each influence factor has its own influence factors so that the number of the overall contributors to the uncertainty of the measurand exceeds 30 , then assigning a normal $p d f$ to the measurand is justified.

\subsection{Continuous probability distribution functions used in metallurgy practice}

In principle, any continuous function defined on an interval $[a, b] \subset R$ can be used as a $p d f$ on condition that:

$$
\int_{a}^{b} f(x) d x=1
$$

For metallurgists, the most useful pdfs other than the normal one are the log-normal, Weibull, Cauchy (Cauchy-Lorentz) and exponential $p d f[6,7]$. The log-normal $p d f$ is used mainly for grain-size data analysis [6]. The mathematical expression of log-normal distribution is derived from normal one by substituting $\ln (x)$ for $x$ as follows:

$$
f(x)=\frac{1}{\sqrt{2 \pi} \ln \sigma_{g}} \cdot \frac{1}{x} \cdot \exp \left[-\frac{\left(\ln x-\ln \mu_{g}\right)^{2}}{2\left(\ln \sigma_{g}\right)^{2}}\right]
$$

where $\mu_{g}$ is the "geometric mean" and $\sigma_{g}$ is the "geometric standard deviation".

The log-normal $p d f$ is proven by empirical facts. Thus, intended used of a log-normal $p d f$ for a specific sample remains at the latitude of the experimentalist.

In the field of metallurgy, the Weibull $p d f$ is used mostly for failure rate or hazard rate estimation. The $p d f$ of a Weibul random variable $X$ is: $[5,8]$

$$
f(x ; \lambda ; k)=\left\{\begin{array}{c}
\frac{k}{\lambda} \cdot \frac{x}{\lambda} \cdot e^{-\left(\frac{x}{\lambda}\right)^{k}} ; x \geq 0 \\
0 ; x<0
\end{array}\right.
$$

where $k>0$ is the shape parameter and $\lambda>0$ is the scale parameter of the distribution.

The Cauchy (Cauchy-Lorentz) function is used successfully to describe the X-ray diffraction or spectral lines profiles which are subjected to homogeneous broadening. The Cauchy function is many times used as a $p d f$ of $X$ variable defined on $\mathrm{R}$, as follows $[5,6,8]$ :

$$
f\left(x, x_{0}, \gamma\right)=\frac{1}{\pi}\left[\frac{\gamma}{\gamma^{2}+\left(x-x_{0}\right)^{2}}\right]
$$

where $x_{0}$ is the peak location and $\gamma$ is the half-width at half-maximum.

As for normal $p d f, f(x ; 0,1)$ is called the standard Cauchy distribution whose $p d f$ is:

$$
f(x ; 0 ; 1)=\frac{1}{\pi\left(1+x^{2}\right)}
$$

The exponential distribution is fitted to describe the random behavior of a process in which events occur independently at a constant average rate. The $p d f$ of the exponential distribution is: 


$$
f(x ; \lambda)=\left\{\begin{array}{c}
\lambda e^{-\lambda x} ; x \geq 0 \\
0 ; x<0
\end{array}\right.
$$

where $\lambda>0$ is the rate parameter.

The mean of an exponential $p d f$ is $\mu=\frac{1}{\lambda}$ while its standard deviation is $\sigma=\frac{\sqrt{2}}{\lambda}=\sqrt{2} \mu$.

The exponential, Cauchy, log-normal and Weibull $p d f s$ were presented very shortly for the sake of the chapter completeness but normal and uniform distributions will be used extensively in the next subchapters.

\section{The probability distribution function of the compound variables}

A random variable $Y$ is defined as a function of other variables $X_{i}, \mathrm{i}=\overline{1}, \bar{n}$, denoted as $Y=f\left(X_{i}\right)$. In this chapter only the functions met in testing practice are considered and expressed as: $Y=a X+b, Y=\left(X_{1}+\ldots X_{n}\right) / n ; Y=X^{2}$ and $Y=\sqrt{X} ; Y=X_{1} / X_{2}$ and $Y=X^{2}{ }_{1} / X^{2}{ }_{2}$.

\subsection{The probability distribution function of the variable $Y=a X+b$}

The simplest case of a compound variable is that of the variable $Y=a X+b$ where $a$ and $b$ are two positive real numbers. Assuming that the $p d f$ of $X$ is defined on $\mathbb{R}$ then the probability probability $P_{Y}(Y \leq y=a X+b)$ is equal with $P_{X}(X \leq x)$. In this instance, the $c d f s$ of $Y$ and $X$ for $Y=a X+b$ have the same value i.e.

$$
F_{Y}(y)=\int_{-\infty}^{y} f_{Y}(u) d u=F_{X}(x)=\int_{-\infty}^{x} f_{X}(v) d v
$$

Substituting the variable $t=a v+b$ for $v$ in Eq.(56)

$$
\int_{-\infty}^{y} f_{y}(u) d u=\int_{\infty}^{Y} \frac{1}{a} f_{x}\left(\frac{t-b}{a}\right) d t
$$

Accordingly,

$$
f_{y}(y)=\frac{1}{a} f_{X}\left(\frac{y-b}{a}\right)
$$

\subsection{The probability density function of linear compound variables}

Consider two random variables $X_{1}$ and $X_{2}: \mathbb{R} \rightarrow \mathbb{R}$. If a variable $X$ is defined on the intervals, it can be considered defined on $\mathbb{R}$ because their $p d f s$ can be extended to $\mathbb{R}$ as follows:

$$
f_{X_{1}}(x)=\left\{\begin{array}{c}
f_{X_{1}} ; x \in[a, b] \\
0 ; x \notin[a, b]
\end{array}\right.
$$

The extension to $\mathbb{R}$ for $f_{X_{1}}(x)$ and $f_{X_{2}}(x)$ is necessary in order to make possible their convolution. The $c d f$ of the variable $Y$ is:

$$
F_{Y}(y)=\mathrm{P}(\mathrm{Y} \leq y)=P_{Y}\left(X_{1} \cup X_{2}\right)
$$

where $P_{Y}\left(X_{1} \cup X_{2}\right)$ is the event, conditioned by $X_{1}+X_{2} \leq y$. If the $p d f s$ of the $X_{1}, X_{2}, Y$ variables are used then the Eq.(60) becomes: 


$$
\begin{gathered}
F_{Y}(y)=\int_{-\infty}^{Y} F_{Y}(t) d t=\int_{x_{1}} \int_{x_{2}} f_{X_{1}}\left(x_{1}\right) \cdot f_{X_{2}}\left(x_{2}\right) d x_{1} d x_{2} \\
\left(\mathrm{x}_{1}+\mathrm{x}_{2}<\mathrm{y}\right)
\end{gathered}
$$

Substituting the variable $u$ for $x_{1}$ and $v$ for $x_{1}+x_{2}$ in Eq.(61)

$$
F_{Y}(y)=\int_{-\infty}^{Y} f_{Y}(t) d t=\int_{-\infty}^{y}\left[\int_{-\infty}^{+\infty} f_{X_{1}}(u) \cdot f_{X_{2}}(v-u) d u\right] d v
$$

Accordingly,

$$
f_{Y}(y)=\int_{-\infty}^{+\infty} f_{X_{1}}(u) f_{X_{2}}(y-u) d u=f_{X_{1}} \otimes f_{X_{2}}(y)
$$

where $f_{Y}(y)$ is the convolution of the of $f_{x_{1}}$ and $f_{x_{2}} p d f s$.

The convolution of two functions is a mathematical operator which has specific properties as commutatively and associatively, but the most important property lies in the fact that the Fourier transform of $f_{X_{1}} \otimes f_{X_{2}}$ is the product of the Fourier transforms of the respective functions.

Based on Eq.(63) one may supposes that the $p d f$ of the variable $Y_{n}(y)=X_{1}+X_{2}+\ldots . X_{n}$ is:

$$
F_{Y_{n}}(y)=f_{X_{1}} \otimes f_{X_{2}} \ldots \ldots \otimes F_{X_{n}}(y)
$$

where: $f_{X_{i}}, i=\overline{1, n}$ are the $p d f s$ of the $X_{i}$ variable.

The validity of Eq.(64) is proved by mathematical induction method. Thus, the above assumption is valid on condition that the $p d f$ of the variable $Y_{n+1}(y)=X_{1}+X_{2}+\ldots . X_{n}+X_{n+1}$ is of the same form as that given by Eq.(64). To prove that, $Y_{n+1}$ is written as:

$$
Y_{n+1}(y)=X_{1}+X_{2}+\ldots . X_{n}+X_{n+1}=Y_{n}+Y_{n+1}
$$

Accordingly,

$$
f_{\mathrm{Yn}+1}(y)=f_{Y_{n}} \otimes f x_{\mathrm{n}+1}(y)=f_{X_{1}} \otimes f_{X_{2}} \cdots \otimes f_{X_{n}} \otimes f_{X_{n+1}}
$$

Consequently, , Eq.(66) proves that Eq. (64) is valid for any $n$. In the case $f_{X}=f_{X}, i=\overline{1, n}$, the $p d f$ of $Y_{n}$ variable is the convolution product of $n$ identical functions, denoted as :

$$
f_{Y_{n}}(y)=f_{X}^{\otimes n}(y)
$$

The $p d f$ of a $Y_{n}$ variable defined as: $Y_{n}=a_{1} X_{1}+a_{2} X_{2}+\ldots \ldots+a_{n} X_{n}$ where $X_{i}, i=\overline{1, n}$ are random variables, $a_{i}, i=\overline{1, n}$ are real numbers, can be calculed in two steps. In the first step, the variables $Z_{i}=a_{i} X_{\mathrm{i}}$ are introduced and then their $p d f s$ are calculated as;

$$
f_{Z_{i}}\left(Z_{i}\right)=\frac{1}{|a|} f_{X_{i}} \cdot\left(\frac{x_{i}}{a_{i}}\right)
$$

Next, $f_{Y_{n}}(y)$ is calculated using the Eq. (64):

$$
f_{Y_{n}}(y)=f_{z_{1}} \otimes f_{z_{2}} \ldots \otimes f_{z_{n}}(y)
$$


Note: The variables: $Y_{n}=\sum_{i=1}^{n} X_{i}$, with $X_{i}=X, i=\overline{1, n}$ and $Y_{n}=n \cdot X$ are diffrent.

The variable $\overline{\bar{X}}_{n}$, assigned to the mean of $\mathrm{n}$ numerical results obtained in repeatability conditions, also called as sample mean variable, is the typical variable to which the linear compound variables theory is applied. Thus, the $\overline{\bar{X}}_{n}$ has the expression:

$$
\overline{\bar{X}}_{n}=\frac{1}{n}\left(X_{1}+X_{2}+\cdots X_{n}\right)=\frac{1}{n} \cdot Y_{n}
$$

where $X_{i}, i=\overline{1, n}$, are the variable assigned to each measurement.

The pdf of $\overline{\bar{X}}$ is:

$$
f_{\overline{\bar{X}}_{n}}(\bar{x})=n \cdot f_{Y_{n}}(\overline{\bar{x}} \cdot n)=n \cdot f_{X}^{\otimes n}(n \cdot \overline{\bar{x}})
$$

If $f_{X}(x)$ is known then the experimental mean distribution can be calculated, and subsequently, the dispersion of the experimental mean around the conventional true mean $\mu$ of $X$ can be estimated as well.

\subsection{The probability density function of $Y=X^{2}$ variable}

Consider a random variable $X$ with $f_{X}: \mathbb{R} \rightarrow \mathbb{R}$ and a variable $Y=X^{2}$. By its definition $Y$ has the following $c d f$ :

$$
F_{Y}(y)=\left\{\begin{array}{c}
0 ; y<0 \\
F_{Y}(y)=\int_{u=0}^{y} f_{Y}(u) d u ; y \geq 0
\end{array}\right.
$$

where $f_{Y}(y)$ is the $p d f$ of $Y$ for $y \geq 0$.

The condition $u \leq y$ implies that $x^{2} \leq y$ i.e $X \in[-\sqrt{y}, \sqrt{y}]$, respectively. Accordingly, the probability that $u \in[0, y]$ is equal to the probability that $X \in[-\sqrt{y}, \sqrt{y}]$ i.e.

$$
P(Y \leq y)=P(-\sqrt{y} \leq x \leq \sqrt{y})=F_{x}(\sqrt{y})-F_{x}(-\sqrt{y})
$$

The Eq.(73) may be expressed as:

$$
\int_{-\sqrt{y}}^{\sqrt{y}} f_{X}(x) d x=\int_{=\sqrt{y}}^{0} f_{X}(x) d x+\int_{0}^{\sqrt{y}} f_{X}(x) d x=I_{1}+I_{2}
$$

where $I_{1}=\int_{x_{1}=-\sqrt{y}}^{x_{2}=0} f_{X}(x) d x, I_{2}=\int_{0}^{\sqrt{y}} f x(x) d x$

Substituting the variable $-\sqrt{t}$ for $X$ into $I_{1}$

$$
I_{1}=\int_{y}^{0} f_{X}(-\sqrt{t})\left(-\frac{1}{2 \sqrt{t}} d t\right)=\frac{1}{2} \int_{0}^{y} \frac{f_{X}(-\sqrt{t})}{\sqrt{t}} d t
$$

Likewise, if in $I_{2}$ is applied the substitutions $\sqrt{t}$, for $x$ then

$$
I_{2}=\frac{1}{2} \int_{0}^{y} \frac{f x(\sqrt{t})}{\sqrt{t}} d t
$$

From Eqs. (74), (75) and (76) it is deduced that: 


$$
f_{Y}(y)=(1 /(2 \sqrt{y})) \cdot[f x(-\sqrt{y})+f x(\sqrt{y})]
$$

The repartition density of $X^{2}$ variable differs significantly from that of $X$. For example, if $X$ is a variable with the $N(0,1) p d f$ then the the $p d f$ of variable $Y=X^{2}$ is:

$$
f_{Y}(y)=\frac{1}{2 \sqrt{y}} y^{-1 / 2} e^{-y / 2}
$$

which is of Weibull type.

\subsection{The probability distribution function of $Y=\sqrt{X}$ variable}

Consider $X$ as a random continuous and positive variable with the $p d f \mathrm{fx}(\mathrm{x}): \mathbb{R} \rightarrow \mathbb{R}$. The $Y=\sqrt{X}$ variable has the value $y=\sqrt{x}$ when $X=x$. The $c d f$ of $Y$ is:

$$
F_{Y}(y)=\int_{0}^{y} f_{Y}(u)=P_{Y}(Y \leq y)
$$

On the other hand, $P_{Y}(Y \leq y)$ is equal to $P_{X}(X \leq x)$ i.e.

$$
\int_{0}^{y} f_{Y}(u) d u=\int_{0}^{x} f_{X}(t) d t
$$

If on the right hand side of Eq.(80) one substitutes $\mathrm{v}^{2}$ for $t$ then

$$
\int_{0}^{y} f_{Y}(u) d u=\int_{0}^{y} 2 v f_{X}\left(v^{2}\right) d v
$$

Accordingly,

$$
f_{Y}(y)=2 y f_{X}\left(y^{2}\right)
$$

Generally, Eq.(79) is used for estimating the $p d f$ of the standard deviation when the $p d f$ of sample dispersion (variance) is known.

\subsection{The probability distribution function of the ratio of two distributions}

Let be the two random and independent variables $X_{1}$ and $X_{2}$ and their $p d f_{s} f_{X_{1}}\left(x_{1}\right)$ and $f_{X_{2}}\left(X_{2}\right)$, respectively defined on $\mathbb{R}$. The variable $Y=X_{1} / X_{2}$ has the repartition function:

$$
F_{Y}(y)=\int_{-\infty}^{y} f_{Y}(t) d t=P_{Y}(Y \leq y)
$$

Substituting $u$ for $x_{2}$ and $u \cdot v$ for $x_{1}$ in Eq.(83)

$$
f_{Y}(y)=\int_{v=0}^{y} \int_{-\infty}^{+\infty} f_{X_{1}}(u \cdot v) \cdot f_{X_{2}}(u) \cdot\left|D\left(\frac{x, y}{u, v}\right)\right| d u d v
$$

where $D\left(\frac{x, y}{u, v}\right)=\left|\begin{array}{ll}\frac{d x_{1}}{d u} & \frac{d x_{1}}{d v} \\ \frac{d x_{2}}{d u} & \frac{d x_{2}}{d v}\end{array}\right|=\left|\begin{array}{cc}v & u \\ 1 & 0\end{array}\right|=-u$ is the Jacobian of the coordinate transformation

From Eqs.(83) and (84)::

$$
f_{Y}(y)=\int_{-\infty}^{+\infty} f_{X_{1}}(u \cdot v) \cdot f_{X_{2}}(u) \cdot\left|D\left(\frac{x, y}{u, v}\right)\right| d u d v
$$


The $p d f$ of $Y=X_{1} / X_{2}$ variable with $X_{1}$ and $X_{2}$ of $N(0,1)$ type is:

$$
f_{Y}(y)=\int_{-\infty}^{+\infty} \frac{1}{\sqrt{2 \pi}} \cdot e^{-\frac{u^{2} y^{2}}{2}} \cdot \frac{1}{\sqrt{2 \pi}} e^{-u^{2}}|u| d u=\frac{1}{\pi\left(1+y^{2}\right)}
$$

Eq.(86) shows that the $p d f$ of the ratio of two variables with standard normal distribution is the Cauchy standard distribution.

\subsection{General approach for deriving the $p d f$ of the sample mean variable}

As reported in literature [2, 8] the sample mean of a sample size " $n$ " i.e. $\left\{x_{1}, x_{2}, \ldots, x_{n}\right\}$ is an estimator for the population mean. On the other hand, the mean has two different meanings: 1) numeric value of the sample mean calculated from observed values of the sample and 2) a function of random variables from a random sample. This subchapter addresses the $p d f$ assigned to the mean of the outcomes of $\mathrm{n}$ repeated tests. Therefore, the mean as a function is a sum of $n$ identical functions divided by $n$. The $p d f$ of $X$, as it was derived in $\S 3.1$, is:

$$
f_{\bar{X}}(\bar{x})=n f^{\otimes n}(n \bar{x})
$$

In the next three paragraphs the $f_{\bar{X}}$ will be deducted for Gaussian, uniform and Cauchy $p d f s$.

\subsection{The probability distribution function of mean of " $n$ " Gaussian variables}

Let $Y_{\mathrm{n}}$ be a compound variable of $n$ identical Gaussian variable $X$ defined as $Y_{n}=$ $X_{1}+X_{2}+\ldots+X_{n}$, where: $X_{i} \equiv X, i=\overline{1, n}$. The $p d f$ of $X$ is:

$$
f_{\left(x ; \mu, \sigma^{2}\right)} \equiv f(x)=\frac{1}{\sigma \sqrt{2 \pi}}=e^{-\frac{(x-\mu)^{2}}{2 \sigma^{2}}}
$$

As proven in $\S 3.1 .2$., the $p d f$ of $Y_{2}=X_{1}+X_{2}$ is:

$$
f_{Y_{2}}(y)=\int_{-\infty}^{+\infty} f_{X_{1}}(y-u) \cdot f_{X_{2}}(u) d u
$$

Eq.(89) shows that the $p d f$ of $Y_{2}$ is of Gaussian type with the mean $\mu_{2}=2 \mu$ and standard deviation $\sigma_{2}=\sqrt{2} \cdot \sigma$. Based on the above result, let's assume that the $Y_{n}$ variable has a $p d f$ of the form

$$
f_{Y_{n}}(y)=\frac{1}{\sqrt{n} \sigma \cdot \sqrt{2 \pi}} \cdot e^{-\frac{(\sigma-n \mu)^{2}}{2 n \sigma^{2}}}
$$

According to the complete induction method the above assumption is true if on the basis of Eq.(90) it can be proven that the $p d f$ of the $Y_{n+1}$ variable is:

$$
f_{Y_{n+1}}(Y)=\frac{1}{\sigma \sqrt{n+1} \cdot \sqrt{2 \pi}} \cdot e^{-\frac{(y-(n+1) \mu)^{2}}{2(n+1) \sigma^{2}}}
$$

The variable $Y_{n+1}=Y_{n}+X_{n+1}$, therefore $p d f$ of $Y_{n+1}$ may be written as;

$$
\frac{1}{2 \pi \sqrt{n} \sigma^{2}} \int_{-\infty}^{+\infty} e^{-\frac{(y-u-\mu)^{2}}{2 \sigma^{2}} \cdot e^{\frac{-(u-n \mu)}{2 n \sigma^{2}}} d u}=\frac{e^{-\frac{(y-(n+1) \mu)^{2}}{2(n+1) \sigma^{2}}}}{\sigma \cdot \sqrt{n+1 \cdot \sqrt{2 \pi}}}
$$


Eq.(92) proves that Eq.(90) is true and permits to state that the $p d f$ of a sum of " $n$ " identical Gaussian variable is of Gaussian type having the mean $\mu_{n}=n^{*} \mu$ and the standard deviation $\sigma_{n}=\sqrt{n} \cdot \sigma$

As it was proven in $\S 3.1$. the $p d f$ of the sample mean variable $\bar{X}$ is:

$$
f_{\bar{x}}(\bar{x})=n \cdot f_{Y_{n}}(n, \bar{x})=n \cdot \frac{1}{\sigma \sqrt{n} \cdot \sqrt{2 \pi}} \cdot e^{\frac{-(n \bar{x}-n \mu)}{2 n \sigma^{2}}}=\frac{-\frac{(\bar{x}-\mu)^{2}}{2 \sigma_{\bar{x}}^{2}}}{\sigma_{\bar{x} \cdot \sqrt{2 \pi}}}
$$

where $\sigma_{\bar{x}}=\sigma / \sqrt{n}$ is the standard deviation of the mean when the sample size is $n$.

Eq.(93) shows that the mean values $(\bar{x})$ are centered on the population mean $\mu$ and their standard deviation is $\sqrt{n}$ times smaller than standard deviation of the population.

The mean and the variance of $X$ could be easily estimated on the base of mean operator $(M)$ and of variance $V$ one applied to a vector of statistical variable, i.e.[6]

$$
\begin{aligned}
& M\left(\sum_{i=1}^{n} a_{i} \cdot X_{i}\right)=a_{i} \sum_{i=1}^{n} M\left(X_{i}\right)=\frac{1}{n} \cdot n \cdot \mu=\mu \\
& V\left(\sum_{i=1}^{n} a_{i} \cdot X_{i}\right)=a_{i}^{2} \sum_{i=1}^{n} V\left(X_{i}\right)=\frac{1}{n^{2}} n \sigma^{2}=\frac{\sigma^{2}}{n}
\end{aligned}
$$

where: $a_{1}=1 / n, i=\overline{1, n}$, but these operations has no meaning for the experimentalist.

\subsection{The probability distribution function of mean of some uniform variable}

In real world testing situations are often found where there is no knowledge about the $p d f$ of the measurand. In such cases, the experimentalists have to consider that the $p d f$ assigned to the measurand is of the uniform type. The same, metallurgical practice implies statistical modeling using additive uniform variable. Simple examples are: weight or length of a chain with $n$ links, strength resistance of a series of $n$ bars, fiability assessment of a product composed of $n$ parts. According to $\$ 2.3 .2$ any updf may be related to a so called standard updf having the width $2, \mu=0$ and $\mathrm{SD}=\sqrt{3} / 3$

Based on the above consideration this section addresses only standard updf given by Eq.(34).

As was derived in 3 . 2.1, the $p d f$ assigned to arithmetic mean of $n$ outcomes is:

$$
f_{\overline{\bar{X}}}=n \cdot f_{X}^{\otimes n}(x)
$$

where $X_{i}=X, i=\overline{1, n}$.The first step in deriving the $p d f$ of $X$ is to calculate the $f_{(X)}^{\otimes n}$. The expression of $f_{(x)}^{\otimes n}$ will be derived by recurrence approach i.e $f^{\otimes((k+1)}=f^{\otimes k} \otimes f(x)$. Thus, the two fold convolution of $\mathrm{f}$ is:

$$
f_{(x)}^{\otimes 2}=\int_{-\infty}^{+\infty} f_{X_{1}}(x-u) \cdot f_{X_{2}}(u) d u=\int_{=\infty}^{+\infty} f_{X_{1}}(u) \cdot f_{X_{2}}(u-x) d u
$$

where $f_{X}(u-x)$ is equivalent with a translation of the graph of $f_{X_{2}}$ with $x$ related to the origin of coordinate. The value of the above integral is proportional to the overlapping area of the graphs of the $X_{1}$ and $X_{2} p d f s$, which is: 


$$
f^{\otimes 2}(x)=\left\{\begin{array}{c}
\frac{1}{2^{2}}(2-|x|) ; x \in[-2,2] \\
0 ; \notin[-2,2]
\end{array}\right.
$$

The $f^{\otimes 2}$ is known and as Simpson distribution [9]. The convolution of two uniform variables gives a triangle distribution with the same height as convoluted $p d f$ but two times larger.

The $f_{(x)}^{\otimes 3}$ is calculated as:

$$
f_{(x)}^{\otimes 3}=\int_{-\infty}^{+\infty} f^{\otimes 2}(u) \cdot f(u-x) d x=\left\{\begin{array}{c}
\frac{1}{2^{3}}\left(3-x^{2}\right) ; x \in[-1,1] \\
\frac{1}{2^{4}}(3-|x|)^{2} ; x \in[-3-1) \cup(1,3] 1 \leq|x| \leq 3 \\
0 ;|x|>3
\end{array}\right.
$$

$f^{\otimes 3}$ is very important because it is the keystone from where the $p d f$ of a sum of identical uniform distribution turns in a polynomial shape (Figure 4). The first order derivative of $f^{\otimes 3}$ is continuous in $X= \pm 1$ but the second order one is not.

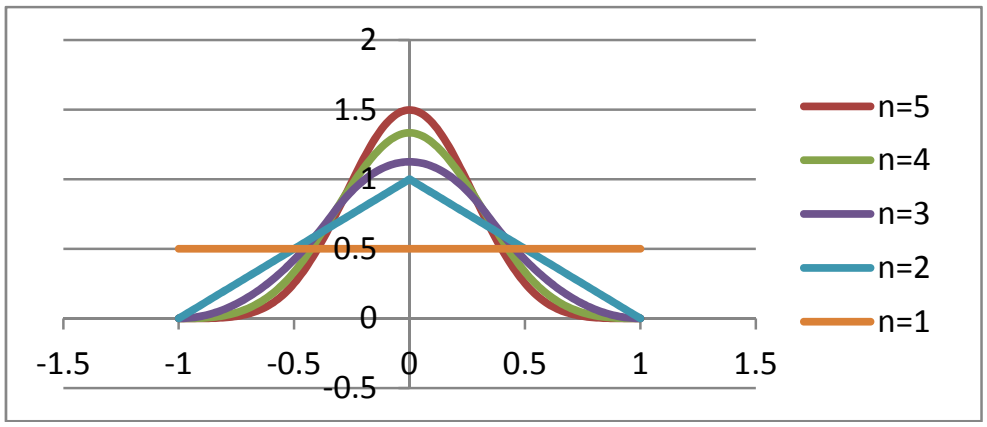

Figure 4. The graphs of the sample means $p d f s$ for $n=\overline{1,5}$

Thus, at $x_{1,2}= \pm 1$ are two inflection points. In the same way as for $f_{(x)}^{\otimes 3}, f^{\otimes 4}$ can be calculate as;

$$
f_{(x)}^{\otimes 4}=\left\{\begin{array}{c}
\frac{1}{2^{4} \cdot 3 !}\left(32-12 x^{2}+3|x|^{3}\right) ;|x|<2 \\
\frac{1}{2^{4} \cdot 3 !}(4-|x|)^{3} ; 2 \leq|x| \leq 4 \\
0 ; x>4
\end{array}\right.
$$

The $f^{\otimes 5} p d f$ is deduced as:

$$
f_{(X)}^{\otimes 5}=\left\{\begin{array}{c}
\frac{2}{2^{5} \cdot 4 !}\left(115-30 x^{2}+3 X^{4}\right) ;|x|<1 \\
\frac{4}{2^{5 \cdot 4 !}}\left(55-10|x|-30 X^{2}+10|x|^{3}-X^{4}\right) ; 1 \leq|x|<3 \\
\frac{4}{2^{5} \cdot 4 !}(5-|x|)^{4} ; 3 \leq|x| \leq 5 \\
0 ;|x|>5
\end{array}\right.
$$


The expressions of the $k$-convolved standard updfs are difficult to be calculated for $k>5$, but one can get help using the general form of a sum of $n$ identical standard uniform variables given by Renyi[9]:

$$
f_{(x)}^{\otimes n}\left\{\begin{array}{cc}
\frac{1}{(n-1) ! 2^{n}} \sum_{i=0}^{\hat{n}(n, x)}(-1)^{1} \cdot C_{n}^{i}(x+n-2 i)^{n-1} ;-n \leq x \leq n \\
0 ; \quad \text { otherwise }
\end{array}\right.
$$

where $\hat{n}(n, x)=\left[\frac{x+n}{2}\right]$ is the largest integer less than $\frac{x+n}{2}$

The $p d f$ of the sample mean of the 5 uniform distributed outcomes obtained in repetitive or reproductive condition, denoted $f_{M 5}(m)=5 f_{(5 m)}^{\otimes 5}$, is

$$
f_{M 5}(m)=\left\{\begin{array}{c}
\frac{10}{2^{5} \cdot 4 !}\left(115-6 \cdot 5^{3} m^{2}+3 \cdot 5^{4} \cdot m^{4}\right) ;|m|<\frac{1}{5} \\
\frac{20}{2^{5} \cdot 4 !}\left(55-50|m|-6 \cdot 5^{3} m^{2}+2 \cdot 5^{4} \cdot|m|^{3}-5^{4} \cdot m^{4}\right) ; \frac{1}{5} \leq|m|<\frac{3}{5} \\
\frac{5^{5}}{2^{5} \cdot 4 !}(1-|m|)^{4} ; \frac{3}{5} \leq|m| \leq 1 \\
0 ;|m|>1
\end{array}\right.
$$

The graphs of the sample means for $n=\overline{2,5}$ are given in Figure 4. A special attention is drawn to $f_{M 5}(m)$ because it is appropriate for the hardness test where the standard recommends five reproductive measurements. Based on the $f_{M 5}$ there were calculated: $P\left(|x|<\frac{1}{5}\right) \cong$ 25,5\%; $P\left(\frac{1}{5} \leq|x|<\frac{3}{5}\right)=44.8 \% ; P\left(\frac{3}{5} \leq|x|<1\right)=0.7 \%$. On the other side, the probability that the mean lies in the interval $\left(-\frac{1}{10}, \frac{1}{10}\right)$ is about $29,5 \%$ while in $\left(-\frac{2}{10},-\frac{1}{10}\right] \cup\left[+\frac{1}{10} ; \frac{2}{10}\right)$ is $25,5 \%$. Thus, the probability that the mean depart from zero decreases relative slowly, not so rapidly as is argued elsewhere [10]. Based on the convolution approach or on Renyi's formula the experimentalist could calculate the $p d f$ of the sample mean for its own $n$ number of repeated tests.

\subsection{The $p d f$ of mean of some Cauchy distributed variable}

Let us consider two Cauchy variable $X_{1}$ and $X_{2}$ whose $p d f s$ defined on $\mathbb{R}$ are:

$$
f_{X_{1}}=\frac{a_{1}}{\pi\left(a_{1}^{2}+x^{2}\right)} \text { and } f_{X_{2}}(x)=\frac{a_{2}}{\pi\left(a_{2}+x^{2}\right)}
$$

The $p d f$ of the variable $Y_{2}=X_{1}+X_{2}$ is:

$$
f_{Y_{2}}(X)=\int_{-\infty}^{+\infty} f x_{1}(x-u) f x_{2}(u)=\frac{a_{1}+a_{2}}{\pi\left[\left(a_{1}+a_{2}\right)^{2}+x^{2}\right]}
$$

In testing practice $X_{1}$ and $X_{2}$ are the same i.e. $a_{1}=a_{2}=a$ therefore $f_{Y_{2}}$ is:

$$
f_{Y_{2}}(x)=\frac{2 a}{\pi\left[(2 a)^{2}+x^{2}\right.}
$$

There expression of $n$-fold convolved Cauchy $p d f s$ is :

$$
f_{Y_{n}}(\mathrm{x})=\frac{n a}{\pi\left[(n a)^{2}+x^{2}\right.}
$$


The $p d f$ assigned to the mean of a set of $n$ outcomes having Cauchy distribution is:

$$
f_{M_{n}}(m)=n \cdot f_{Y_{n}}(n m)=\frac{n^{2} a}{\pi(n a)^{2}+(n m)^{2}}=\frac{a}{\pi\left(a^{2}+m^{2}\right)}
$$

Eq.(108) shows that the pdf of mean is identical with that of the measurand. As a paradox, repeating a test many times on a measurand whose $p d f$ is of Cauchy type is of no use. Since the dispersion of the Cauchy distribution is infinity then it should be avoided being assigned to a measurand.

\subsection{Student's or t-distribution}

The Student's distribution, also referred to as t-distribution, is used on a large scale to test the exactness of a set of numerical outcomes of repeated tests when $\mu$ and $\sigma$ are not known. The power of t-tests consists in making use of $\mu$ and $\sigma$ as hidden or implicit variable for statistical inference while they remain unknown. Thus, let be a set of $\mathrm{n}$ outcomes $\left\{x_{1}, x_{2}, \ldots, x_{n}\right\}$ whose mean $\bar{x}$ and sample dispersion $s^{2}$ are, respectively

$$
\begin{gathered}
\bar{x}=\frac{1}{n} \sum_{i=1}^{n} x_{i} \\
s^{2}=\frac{1}{n-1} \sum_{i=1}^{n}\left(x_{i}-\bar{x}\right)^{2}
\end{gathered}
$$

The experimentalist is concerned about the exactness of $\bar{x}$, i.e. the accuracy $\bar{x}-\mu$ related to the standard deviation of mean $s_{\bar{x}}=s / \sqrt{n}$. Thus, the parameter $t=(\bar{x}-\mu) / s_{\bar{x}}$ was found to be the best estimator for the case of a test with unknown $\mu$ and $\sigma$. Fortunately, a t-parameter can be written as:

$$
t=\left(\frac{\bar{x}-\mu}{\frac{\sigma}{\sqrt{n}}}\right) /\left(\frac{\frac{s_{\bar{x}}}{\sqrt{n}}}{\frac{\sigma}{\sqrt{n}}}\right)=\left(\frac{\bar{x}-\mu}{\sigma_{\bar{x}}}\right) /\left(\frac{S_{\bar{x}}}{\sigma_{\bar{x}}}\right)
$$

The $p d f$ of the variable $T$ assigned to $t$ values will be derived as the ratio of the variables $Z$ assigned to $(\bar{x}-\mu) / \sigma_{\bar{x}}$ and $\mathrm{R}$ to $\left(s_{\bar{x}} / \sigma_{\bar{x}}\right)$. The $Z$ variable has a $p d f$ of $N(0,1)$ type while the $p d f$ of $R$ will be defined a little bit later. Before proceeding to derive the expression of the $p d f$ of $R$ variable, let make some reconsiderations about the actual way of deriving the $p d f$ of $S^{2}$. Thus, on the basis of the well known Eq.(110) the variable $S^{2}$ assigned to $s^{2}$ is considered as:

$$
S^{2}=\frac{(n-1)}{\sigma^{2}} Y_{n}^{2}
$$

where $Y_{n}^{2}=\left(X_{1}-\mu\right)^{2}+\cdots+\left(X_{n}-\mu\right)^{2}$

Here it is considered that the above issue is quite unproductive because the $(n-1)$ factor replaces the $n$ factor in Eq.(107) just because $s n^{2}$ approximates better the sample variance related to $\mu$. i.e.

$$
\frac{1}{n-1} \sum\left(x_{i}-\bar{x}\right)^{2} \cong \frac{1}{n} \sum_{i=1}^{n}\left(x_{i}-\mu\right)^{2}=\frac{\sigma^{2}}{n} \sum_{i=1}^{n}\left(\frac{x_{i}-\mu}{\sigma}\right)^{2}
$$

Therefore the variable attached to $S^{2}$ should be: 


$$
S^{2}=\frac{\sigma^{2}}{n} \sum_{i=1}^{n} \phi_{i}\left(n_{i}\right)=\frac{\sigma^{2}}{n} \phi_{n}
$$

where $\phi_{i}=N(0,1), i=\overline{1, n}$ and $\phi_{n}=\sum_{i=1}^{n} \phi_{i}$.

The $p d f$ of $\phi_{n}$, as it was shown in $\S 3.3$.), is:

$$
f_{\phi_{n}}(u)=\frac{u^{\frac{n}{2}-1} e^{-\frac{n}{2}}}{2^{\frac{n}{2}} \Gamma\left(\frac{n}{2}\right)}
$$

The $p d f$ of $S_{\mathrm{n}^{2}}$ variable is:

$$
f_{S_{n}{ }^{2}}(w)=\frac{n}{\sigma^{2}} f_{\phi_{n}}\left(\frac{n w}{\sigma^{2}}\right)=\frac{n}{\sigma^{2}} \frac{\left(\frac{n w}{\sigma^{2}}\right)^{\frac{n}{2}-1} e^{-\frac{n w}{2 \sigma^{2}}}}{2^{\frac{n}{2}} \Gamma\left(\frac{n}{2}\right)}
$$

The pdf of $S_{n}=\sqrt{S_{n}{ }^{2}}$ variable is:

$$
f_{S_{n}}(s)=2 s \cdot f_{S_{n}}{ }^{2}\left(s^{2}\right)=\frac{2 s\left(\frac{n}{\sigma^{2}}\right)\left(\frac{n s^{2}}{\sigma^{2}}\right)^{\frac{n}{2}-1} e^{-\frac{n s^{2}}{2 \sigma^{2}}}}{2^{\frac{n}{2}} \Gamma\left(\frac{n}{2}\right)}
$$

The $p d f$ of the variable $R=S_{n} / \sigma$ is:

$$
f_{R}(r)=\sigma f_{S_{n}}(r \sigma)=\frac{2 n r(n)^{\frac{n}{2}-1} r^{n-2} e^{-\frac{n r^{2}}{2}}}{2^{\frac{n}{2}} \Gamma\left(\frac{n}{2}\right)}=\frac{2 n^{\frac{n}{2}} r^{n-1} e^{-\frac{n r^{2}}{2}}}{2^{\frac{n}{2}} \Gamma\left(\frac{n}{2}\right)}
$$

The $p d f$ of $T=\varnothing_{n} / R$, according to $\S 3.1$, can be calculated as:

$$
f_{T}(t)=\int_{0}^{\infty} \frac{e^{-\frac{(t u)^{2}}{2}}}{\sqrt{2 \pi}} \frac{2 n^{\frac{n}{2}} u^{n-1} e^{-\frac{n u^{2}}{2}}}{2^{\frac{n}{2}} \Gamma\left(\frac{n}{2}\right)} u \cdot d u=\frac{n^{\frac{u}{2}}}{\sqrt{2 \pi} 2^{\frac{n}{2}} \Gamma\left(\frac{n}{2}\right)} \int_{0}^{\infty} e^{-\frac{u^{2}\left(t^{2}+n\right)}{2}} u^{n-1} 2 u \cdot d u
$$

Substituting $x$ for $\left[u^{2}\left(t^{2}+n\right)\right] / 2$ in Eq.(119)

$$
f_{T}(t)=\frac{n^{\frac{n}{2}}}{\sqrt{2 \pi} 2^{\frac{n}{2}} \Gamma\left(\frac{n}{2}\right)} \int \frac{x^{\frac{n-1}{2}} 2^{\frac{n-1}{2}} e^{-x}}{\left(t^{2}+n\right) \frac{n-1}{2}} \frac{1}{\left(t^{2}+n\right)} \cdot d x=\frac{\Gamma\left(\frac{n+1}{2}\right)}{\sqrt{\pi n} \Gamma\left(\frac{n}{2}\right)}\left(1+\frac{t^{2}}{n}\right)^{\frac{-(n+1)}{2}}
$$

The expression of $f_{T}(t)$ is consistent with those given in different works [6, 8] if $n$ is replaced by $v$ which is the so called number of degrees of freedom $(n d f)$ of the variable under consideration.

The derivation of $f_{T}(t)$, frequently denoted as $f(t)$, is important for two reasons: a) the Student's $p d f$ must be applied only for a set of identical Gaussian variables or $n$ repeated test when the $p d f$ of the measurand is of normal (Gaussian) type; b) to correctly establish the $n d f$.

The $c d f$ of $T, F_{\mathrm{T}}(t)$, cannot be estimated as analytical primitive of $f_{T}(t)$, but its values were tabulated and can easily be found in the open literature $[5,6,8]$.The arguing that $v=n$ is very important when $n$ is $2,3,4$ because the $t_{n}(0 ; 0.05)$ varies significantly in this range. Thus, it is a matter of evidence that many times 3 repeated measurements are considered to be enough. 
In this case, using $t_{2}(0.005)=4.303$ instead of $t_{3}=(0.05)=3.182$ increases the expanded uncertainty significantly.

\subsection{Fisher-Snedecor distribution}

The proficiency testing (PT) is a well defined procedure used for estimating the performances of the collaborative laboratories [11, 12]. ANOVA (ANalysis Of VAriance) is one of the methods used for analysis of sample variances reported by the laboratories. ANOVA is based on Fisher-Snedecor distribution of the two sample variances of a measurand $X$. Thus, if for the same sample a laboratory labeled A, gives a sample dispersion.

$$
s_{A}^{2}=\frac{1}{n_{1}-1} \sum_{i=1}^{n_{1}}\left(x_{i}-\overline{x_{1}}\right)^{2}
$$

while B laboratory gives:

$$
s_{B}^{2}=\frac{1}{n_{2}-1} \sum_{j=1}^{n_{2}}\left(x_{j}-\overline{x_{2}}\right)^{2}
$$

where $x_{i}, i=\overline{1, n_{1}}$ and $x_{j}=\overline{1, n_{2}}$ are the outcomes obtained for the same $X$ measurand by the laboratories $\mathrm{A}$ and $\mathrm{B}$, respectively.

As it was argued in $\S 3.4$, to the $s_{A}{ }^{2}$ and $s_{B}{ }^{2}$ may be assigned the variables $S_{A}{ }^{2}$ and $S_{B}{ }^{2}$ whose $p d f s$ are, respectively:

$$
\begin{aligned}
& f_{S_{A}{ }^{2}}(u)=\frac{n_{1} \frac{n_{1}}{2} \cdot u^{\left(\frac{n_{1}}{2}-1\right)} \cdot e^{-\frac{n_{1} u}{2 \sigma^{2}}}}{\sigma n_{1} \cdot 2^{\frac{n_{1}}{2}} \cdot \Gamma\left(\frac{n_{1}}{2}\right)} \\
& f_{S_{B}{ }^{2}}(v)=\frac{n_{2}{ }^{\frac{n_{2}}{2}} \cdot v^{\left(\frac{n_{2}}{2}-1\right)} \cdot e^{-\frac{n_{2} v}{2 \sigma^{2}}}}{\sigma n_{2} \cdot 2^{\frac{n_{2}}{2}} \cdot \Gamma\left(\frac{n_{2}}{2}\right)}
\end{aligned}
$$

The $p d f$ of the variable ratio $S^{2}{ }_{A} / S^{2}{ }_{B}=F$ is:

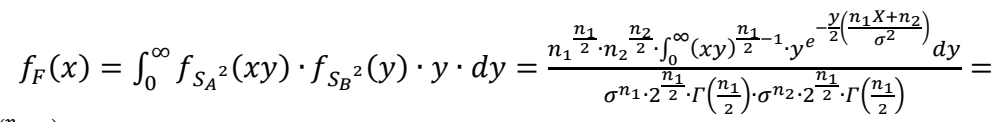

$$
\begin{aligned}
& \frac{n_{1}^{\frac{n_{1}}{2}} \cdot n_{2} \frac{n_{2}}{2} \cdot x^{\left(\frac{n_{1}}{2}-1\right)}}{\sigma^{n_{1}+n_{2}} \cdot 2^{n_{1}+n_{2}} \cdot \Gamma\left(\frac{n_{1}}{2}\right) \cdot \Gamma\left(\frac{n_{2}}{2}\right)} \cdot \int_{0}^{\infty} y^{\frac{n_{1}+n_{2}}{2}-1} \cdot e^{-y\left(\frac{n_{1} x+n_{2}}{2 \sigma^{2}}\right)} d y
\end{aligned}
$$

If the variable $y$ is replaced by $u=y\left(n_{1} x+n_{2}\right) / 2 \sigma^{2}$ in Eq.(125) then:

$$
\begin{aligned}
& f_{F}(x)=\frac{n_{1}^{\frac{n_{1}}{2}} \cdot n_{2}^{\frac{n_{2}}{2}} \cdot x^{\left(\frac{n_{1}}{2}-1\right)}}{\sigma^{n_{1}+n_{2} \cdot 2^{n_{1}+n_{2}} \cdot \Gamma\left(\frac{n_{1}}{2}\right) \cdot \Gamma\left(\frac{n_{2}}{2}\right)}} \cdot \frac{2^{\frac{n_{1}+n_{2}}{2}} \sigma^{\left(n_{1}+n_{2}\right)}}{\left(n_{1} X+n_{2}\right)^{\frac{n_{1}+n_{2}}{2}}} \cdot \int_{0}^{\infty} u^{\frac{n_{1}+n_{2}}{2}-1} e^{-u} d u=\frac{\left(\frac{n_{2}}{n_{1}}\right)^{\frac{n_{1}}{2}} \cdot \Gamma\left(\frac{n_{1}+n_{2}}{2}\right)}{\Gamma\left(\frac{n_{1}}{2}\right) \cdot \Gamma\left(\frac{n_{2}}{2}\right)} \cdot \frac{x^{\frac{n_{1}}{2}-1}}{\left(x+\frac{n_{2}}{n_{1}}\right)^{\frac{n_{1}+n_{2}}{2}}}= \\
& \left(\frac{n_{2}}{n_{1}}\right)^{\frac{n_{1}}{2}} \cdot B\left(\frac{n_{1}}{2}, \frac{n_{2}}{2}\right) \cdot x^{\frac{n_{1}}{2}-1} \cdot\left(x+\frac{n_{2}}{n_{1}}\right)^{-\frac{n_{1}+n_{2}}{2}}
\end{aligned}
$$

The derived expression for $f_{F}(x)$ is identical to that given by Cuculescu, I., [5]. Herein, again arises the problem where $n d f$ is $n-1$ or $n$. By my opinion $v=n$. The $c d f$ of Fisher-Snedecor 
distribution is not an elemental analytical form, but its value for different significance level $(s l)$ and different $n_{1}, n_{2}, F\left(s l, n_{1}, n_{2}\right)$, are tabulated and can be easily found in open literature [5, $6,8]$. The way of using Fisher-Snedecor distribution as F-test consists of comparing the obtained value of $s^{2} A_{A} / s_{B}^{2}=f_{e}$ with the value of $F\left(s l ; n_{1}, n_{2}\right)$ taken from a Fisher $c d f$ table. If $f_{e} \leq F\left(s l ; n_{1}, n_{2}\right)$, then the $s^{2} A$ and $s^{2} B$ are consistent, otherwise one of them is a straggler or an outlier.

Note. A straggler should be considered a correct item, but a statistical outlier is discharged [11].

\section{Measurement uncertainty estimation}

\subsection{General concepts regarding measurement uncertainty estimation}

Apparently the terms "test result" and "measurement result" have the same meaning i.e. a numeric outcome of a measurement process. But in metrology a measurement is defined as a "set of operations having the object of determining a value of a quantity" $[13,14]$ while a test is defined as a "technical operation that consists of the determination of one or more characteristics of a given product, process or service according to a specific procedure" [14]. Thus, a test is a measurement process well documented, fully implemented and permanently supervised. In a test process, the environmental and operational conditions will either be mentioned at standard values or be measured in order to apply correction factors and to express the result in standardized conditions. Besides the rigorous control of the "test conditions", a test result bears an intrinsic inaccuracy depending on the nature of the measurand, on the performance of the method and on the performances of the equipment. Thus, the entire philosophy of the metrology is based on the fact that the true value of the measurand remains unknown to a certain extent when it is estimated based on a set of test outcomes. In this sense, the measurement uncertainty $(M U)$ is defined as an interval about the test result, which may be expected to encompass a large fraction of values that could reasonably be attributed to the measurand. The half width of this interval is called expanded uncertainty, denoted by $U$ or $U(x x)$, where $x x$ is the index of the level of confidence associated to the interval. The confidence level is frequently $95 \%$ but may be about $99 \%$ or $68 \%$ depending on the client requirements or on the test performances. According to EN ISO/IEC 17025 a testing laboratory should apply a procedure to estimate the $M U$ [1]. In a similar manner, when estimating the $M U$ all relevant uncertainty components in a given situation should be taken into account using the appropriate method of analysis. The sources of uncertainty include but are not limited to the reference standards and reference materials used, the method and equipment used, the environmental conditions, the properties and conditions of the item being tested and the operator. The degree of rigor of the uncertainty estimation depends on many factors as those above, but the test result quality is better as the $M U$ is smaller. Thus, the $M U$ is the most appropriate quantitative parameter for test result quality evaluation. As a consequence, the approaches of $M U$ estimation were the subject to different committees of the renowned organizations such as ISO, ILAC, EA, EURACHEM, AIEA etc. Among the $M U$ estimation approaches, the 
one of ISO given in GUM [2] may be considered as the master one. Accordingly, consistency with GUM is generally required for any particular procedure for $M U$ estimation. The basic concepts of GUM are:

1. The knowledge about any influence factor of the measurand is in principle incomplete, therefore its action shall be considered stochastic following a $p d f$;

2. The expected value of the $p d f$ is taken as the best estimate of the influence factor;

3. The standard deviation of the $p d f$ is taken as the best estimate of the standard uncertainty associated to that estimate;

4. The type and the parameter(s) of the $p d f$ have been obtained based on prior knowledge about the influence factors or by repeated trials of the test process.

The $M U$ estimation procedure consists of two main steps. The first step, called formulation, consists of measurand description (physical and mathematical modeling), statistical modeling, input-output modeling and, finally, assigning a $p d f$ to the measurand. The second step, called calculation, consists of deriving the $p d f s$ for the test result estimation (mean, standard derivation, etc.) and the formulas or the algorithm for estimating the $M U$ attributed to the test result.

\subsection{Uncertainty estimation according to GUM}

\subsubsection{Formulation step}

The formulation begins with the measurand definition. The $X$ measurand may be classified as directly accessible to the measure or indirectly measured. The directly measurable measurands as length, mass, temperature etc. are not addressed here but those indirectly measured as elemental concentrations, Young modulus, hardness etc. Anyhow, a SI unit of measure shall be assigned to each measurand whenever it is possible, and also a reference for traceability. The indirect measurand may have a deterministic mathematical model as for Young modulus

$$
E=\frac{F \cdot l_{0}}{S \cdot D l}
$$

where $E$ is the Young modulus, $F$ is the applied force $(\mathrm{N}), S$ is the area $\left(\mathrm{m}^{2}\right), l_{0}$ is the initial length of the specimen, $\Delta l$ is the elongation (m).

Generally, the deterministic model of a measurand $Y$ which is estimated based on the values of a set of measurands $X_{i}, i=\overline{1, n}$ is a function that described the relationship between outcome size and sizes of the inputs expressed as:

$$
Y=f\left(X_{1}, X_{2}, \ldots, X_{n}\right)
$$

Eq.(125) is called the input-output model of the test process. A particular value $y$ of $Y$ is calculated based on the $x_{\mathrm{i}}$ values of $X_{i}: i=\overline{1, n}$ as:

$$
y=f\left(x_{1}, x_{2} \ldots . x_{n}\right)
$$


But, from the statistical point of view the mathematical (statistical) model of the measurand $Y$ is $[11,13]$ :

$$
\mathrm{y}=\mu+\mathrm{b}+\mathrm{e}
$$

where $\mu$ is the general mean or the true value of $Y, b$ is the systematic error or bias and $e$ is the random error occurring in every measurement.

The bias $b$ may be detected and corrected by statistical means. The random error $e$ is caused by the whole set of the influence factors of the input measurands that form the so called uncertainty budget. The $X_{i}, i=\overline{1, n}$ measurands may be directly accessible to the measurement or may not but each $X_{i}$ has its own uncertainty budget. Thus, the statistical model of the $X_{i} ; i=1, n$ are of the form:

$$
X_{i}=\mu_{i}+b_{i}+e_{i}
$$

where $\mu_{i}$ is the test value of $X_{i}, b_{i}$ is the bias of $X_{i}$ and $e_{\mathrm{i}}$ is the random error of $X_{i}$

Thus, the systematic errors and the random ones of the input measurands $X_{i}: i=\overline{1, n}$ are incorporated in the overall uncertainty of the output measurand $Y$. Each $X_{i}: i=\overline{1, n}$ has an uncertainty budget $(U B)$ containing $n_{\mathrm{i}}$ influence factors $F_{i j}, i=\overline{1, n}$, denoted as $U B X_{i}=$ $\left\{F_{i j}\right\} ; j=\overline{1, n_{l}}$. In this instance, the uncertainty budget of $Y$, denoted as $U B Y$, could be expressed as:

$$
U B_{Y}=\cup_{i=1}^{n}\left(U B_{X_{i}}\right)
$$

Eq.(132) shows that the design of an accurate uncertainty budget for a measurand needs advanced knowledge and extended data about the input measurands that, many times, are quite impossible to be achieved. In this context, the $p d f$ of $Y$ remains the most appropriate target for the experimentalist. The $p d f$ assigned to the $Y$ may be of Gaussian type if the influence factors are all of the Gaussian type or if its uncertainty budget contains more than 30 uncorrelated influence factors. Otherwise, to the $Y$ shall be assigned a $p d f$ having a less clustering tendency than the normal one. Same $p d f s$ having less clustering tendency could be ordered as uniform, trapezoidal, Cauchy, etc. The assigning of an appropriate $p d f$ to a measurand is a difficult task which has to be solved by the experimentalist. One of the arguments that underpins the previous affirmation consists the evidence that each measurand has its own variability which is enlarged by the testing process.

\subsubsection{The calculation step}

Suppose that the $p d f$ of a measurand $X, f_{X}(x)$, is established. As it was shown in $\S 3.1$, the arithmetic mean of a set of test results carried on in repetitive or reproductive conditions is the best estimator for the conventional true value of the measurand. The variable assigned to the mean, $M$, is described by a linear model as:

$$
M=\left(X_{1}+X_{2}+\ldots \ldots X_{n}\right) / n
$$


where $x_{\mathrm{i}}, i=\overline{1, n}$, are the statistical variable assigned to each repeated measurement.

The mean is a statistic $[2,5,6$,$] with a p d f$ obtainable by a convolved product as it is shown in $\S 3.2$

$$
f_{M}(y)=n f^{\otimes n}(n y)
$$

Once having the $f_{M}(y)$ then it is quite easily to calculate the probability $p(|\bar{x}-\mu|) \leq \varepsilon$ i.e. to estimate the level of confidence for $\bar{x}-\varepsilon \leq \mu \leq \bar{x}+\varepsilon$.

On the other hand, the design of the mathematical model of the measurand and, subsequently, of the sample mean needs substantial scientific efforts and costs that may be prohibitive. As a consequence, many times laboratories adopt an alternative approach based on prior empirically achieved information.

\subsection{The empirical estimation of $M U$}

If a testing laboratory does not have a mathematical model as a basis for the evaluation of $M U$ of the test results then it has to implement an empirical procedure for $M U$ estimation. The flowchart of such a procedure is a stepped one [3]. Thus, the first step consists of listing those quantities and parameters that are expected to have a significant influence on the test result. Subsequently, the contribution of each influence factor to the overall $M U$ is assessed. Based on the level of contribution to the overall $M U$, each factor shall be classified as significant or irrelevant. The irrelevant influence factors are discarded from the list. The equipment, the CRMs, the operator are among the most frequently considered as significant influence factors. If there is a lack of knowledge or prior data about an influence factor then it is strongly recommended to the laboratory to perform specific measurements to evaluate the contribution of that factor. If the contribution of an influence factor to the overall $M U$ is estimated based on a set of outcomes whose variability is assigned exclusively to that factor then its contribution to the overall $M U$ is considered of the A type. Else its contribution is of type B.

\subsection{Combined uncertainty calculation}

The combined uncertainty incorporates the contributions of the influence factors $F_{i}, i=\overline{1}, n$, to the overall $M U$ i.e. uncertainties of the A type and of B type. The calculus of the combined uncertainty is based on error propagation law $[2,4,8]$. Metallurgical testing practice shows that the influence factors are about always considered as independent. Thus, the combined uncertainty is calculated as:

$$
u_{c}^{2}=u_{A}^{2}+u_{B}^{2}
$$

where $u_{A}$ and $u_{B}$ are the combined uncertainty of A type and of B type, respectively.

The combined uncertainty of the type A is calculated as:

$$
u_{A}=\left(\sum_{i=1}^{n} u_{A_{i}}^{2}\right)^{1 / 2}
$$


where $u_{A i}$ is the uncertainty of type A assigned to the influence factor $F i_{j}, i=\overline{1, n}$.

The combined uncertainty of the type B is calculated as:

$$
u_{B}=\left(\sum_{j=1}^{m} u_{B_{i}}^{2}\right)^{1 / 2}
$$

where $u_{B i}$ is the uncertainty of type $\mathrm{B}$ assigned to the influence factor $F_{j}, j=\overline{1, m}$.

\subsection{Estimation of the expanded uncertainty}

The degree of confidence associated with the combined uncertainty $\left(u_{c}\right)$ is considered, many times, as incompetent, therefore the uncertainty attributed to the measurand is increased to a certain extent in order to comply with a previously stated confidence level, usually $95 \%$. The increased uncertainty is called expanded uncertainty and is usually obtained by multiplying $u_{c}$ with a factor $k$, called coverage factor. For example, the $k$ factor for a measurand with Gaussian $p d f$ is 2 for a $95 \%$ confidence level, i.e. the expanded uncertainty for the $95 \%$ confidence level is $U=2 u_{c}$. The value of $k$ for a specific confidence level depends on the $p d f$ type of the measurand and on the number of test results that were used to calculate the sample mean and sample standard derivation. If the $p d f$ of the measurand is Gaussian or the number of test results exceeds 30 then it is justified to consider that the mean is normally distributed. If the $p d f$ of the measurand is Gaussian but $n<30$ then the Students' $\mathrm{t}$-distribution shall be used.

According to [10,14], in many cases the uniform distribution may be assigned to the measurand. In this case, $U(95)$ is calculated as:

$$
U(95)=1,65 \cdot u_{c}
$$

where $u_{c}$ is the combined uncertainty of the test result

\subsection{Reporting the test result}

The test process yields a value as the estimate for the conventional true value of the measurand. In principle, this value is the sample mean $\bar{y}$ or simply $y$. As standards strongly recommend $[1,2,4]$, the $y$ value must be reported together with its expanded (extended) uncertainty $U$ for a specific confidence level (typically 95\%) as follows:

$$
y \pm U
$$

The laboratory may specify the $p d f$ type and $k$ value as substitutes for the confidence level specification. If in a testing situation a laboratory could not evaluate a metrological sound numerical value for each component of $M U$ then this laboratory may report the standard uncertainty of repeatability, but this shall be clearly stated as it is recommended in $[3,14]$.

The laboratory practice shows that $M U$ estimation is in many cases a time-consuming and costly task. This endeavor shall be justified by the advantage of $M U$ evaluation for testing laboratories. EA 4/16 argues that $M U$ assists in a quantitative manner important issues such 
as risk control, credibility of the test results, competitiveness by adding value and meaning to the test result etc. But, in the author's opinion, the $M U$ estimation imposes the operator increased awareness about the influence factors and higher interest in the means to improve the quality of the test results.

\section{Uncertainty estimation for Rockwell C hardness test. Case study}

\subsection{Hardness testing-general principles}

Hardness is the measure of how resistant a solid bulk material is against ingression by spherical, pyramid or conical tips. Hardness depends on the mechanical properties of the sample as: ductility, elasticity, stiffness, plasticity, strain, strength, toughness etc [15]. On the other hand, quantity "hardness" cannot be expressed as a numeric function of a combination of some of these properties. Therefore, hardness is a good example of measurand that cannot be mathematically modeled without referring to a method of measurement $[4,15]$. In this view, there is a large number of hardness testing methods available (e.g. Vickers, Brinell, Rockwell, Meyer and Leeb) $[15,16]$. As a consequence, there is not a measure unit for hardness independent of its measurement method. In time, based mainly on empirical data, standard methods for hardness testing appropriate to the material grades were developed. In the metallurgical testing field the Brinell, Rockwell and Vickers methods are the most frequently used. For this reason, this case study addresses the well-known Rockwell C hardness by measuring the depth of penetration of an indenter under a specific load. There are different Rockwell type tests denotes with capital letter $\mathrm{A} \div \mathrm{H}[16,17]$, but herein referred only C type.

\subsection{Description of the measurand}

The Rockwell $\mathrm{C}$ hardness is the measure of how resistant a solid bulk material is against penetration by a conical diamond indenter, having a tip angle of $120^{\circ}$, which impinges on the flat surface of the sample with a prescribed force.

The Rockwell C hardness scale is defined as:

$$
h_{R C}=100 x(0.002)-d
$$

where $d$ is the penetration depth.

The most meaningful measure for metallurgists is the Rockwell C index, defined as:

$$
H R C=h_{R C} / 0,002
$$

\subsection{Description of the test method}

The method used for Rockwell C is given in ISO 6805 standard [16]. The Rockwell C test is performed on bulk sample having geometrical dimensions complying with the ISO 6805 specifications. The indenter is diamond cone having the tip angle of $120^{\circ} \pm 0.5^{0}$. The tip ends 
with a hemisphere of $0.002 \mathrm{~mm}$ in diameter. The indentation is a stepwise process i.e. in the first step the indenter is brought contact with the sample surface and loaded with a preliminary test forces $F_{0}=98 \mathrm{~N}$ with a dwell time of $1-3 \mathrm{~s}$. Subsequently, during a period of $1 \mathrm{~s}-8 \mathrm{~s}$, an additional force $F_{1}=1373 \mathrm{~N}$ is loaded. The resulting force $F=1471 \mathrm{~N}$ is applied for $4 \pm 2 \mathrm{~s}$. In the third step the $F_{1}$ force is released and the penetration depth $h$ is measured in the period 3s-5s. The ISO 6805 recommends that the number of tests carried on in repetitive (reproductive) conditions to be a multiple of 5 . In this instance, 5 indentations are established as practice for estimating the conventional true value of HRC of the specimen that has undergone the test. The recommended environmental conditions of the test are [16]: temperature $10-35^{\circ} \mathrm{C}$, protection against: vibrations, magnetic and electric fields, avoiding the soiling of the sample.

\subsection{The mathematical model of the measurand}

The mathematical model given by GUM for estimating $M U$ of the hardness reference blocks is the most appropriate for the $M U$ estimation in the industrial hardness testing. The mathematical model given by GUM takes into account the correction factors coming from equipment calibration as follows:

$$
h_{\mathrm{RC}}=f(\bar{d} ; \Delta c ; \Delta b ; \Delta e)=100 \times 0.002-\bar{d}-\Delta_{c}-\Delta_{b}-\Delta_{e}(\mathrm{~mm})
$$

where $\bar{d}$ is the mean of the penetration depth; $\Delta_{c}$ is the correction assigned to the equipment ; $\Delta_{b}$ is the difference between the hardness of the areas indented by reference equipment and the calibrated area; $\Delta_{e}$ is the correction assigned to the uncertainty of the reference equipment.

$\Delta_{c}$ is assigned to the equipment by comparing its penetration depths, done in a primary hardness reference block, with those of a reference equipment („national etalon machine”).

Although $\Delta_{b}$ and $\Delta_{e}$ are negligible corrections, they are introduced in the expression of the mathematical model to be taken into account as contributors to the $M U$ budget. According to ISO 6508-1:2005 [16] a "Hardness Reference Block” may be considered as CRM (Certified Reference Materials). Based on the above model it was considered that the $h_{R C X}$ of a $X$ specimen may be expressed as:

$$
h_{\mathrm{RCX}}=f(\bar{d} ; \Delta c ; \Delta b ; \Delta e)=100 \times 0.002-\bar{d}_{X}-\Delta_{C}(\mathrm{~mm})
$$

where $\bar{d}_{X}$ is the mean of the penetration depths given by the testing machine on $X$ specimen; $\Delta_{c}$ is the correction assigned to the equipment resulted by comparring the mean penetration depth done on a CRM with those specified by its certificate i.e.

$$
\Delta_{C}=d_{o C R M}-\bar{d}_{C R M}
$$

where $\bar{d}_{C R M}$ is the mean of the penetration depth given by the test machine on CRM, $d_{o C R M}$ is the expected penetration depth derived as:

$$
d_{o C R M}=\left(100-H R C_{C R M}\right) * 0.002
$$


where HRC-CRM is the certified Rockwell C hardness of the CRM

\subsection{Estimation of the contribution of the influence factors to the overall $M U$}

\subsubsection{Contribution assigned to the mean indented depth on specimen $\left(\bar{d}_{X}\right)$}

The contribution of $\bar{d}_{X}$ to the averall $M U$ is assessed by the standard deviation of the mean which is a standard uncertainty (type A). In addition to the data spreading around $\bar{d}_{X}$, the uncertainty of the resolution of depth measuring system will be considered. The contribution of $\bar{d}_{X}$ to the overall $M U$ is:

$$
u_{c}^{2}\left(\bar{d}_{X}\right)=t^{2} \frac{S_{X}^{2}}{n}+\frac{\delta^{2}}{12}
$$

where $S_{X}^{2}$ is the sample variance of the identation depths, $n$ is the number of indentations, $t$ is the Student factor, $\delta^{2} / 12$ is uncertainty assigned to depth reading having a triangle $p d f$ [2] The $S_{X}^{2}$ can be calculated as:

$$
S_{X}^{2}=\frac{1}{n-1} \sum_{i=1}^{n}\left(d_{i}-\bar{d}_{X}\right)^{2}
$$

where $d_{i}$ are the indentation depth, $i=\overline{1, n} ., n$ is the number of indentations

\subsubsection{Contribution assigned $\Delta_{c}$}

The uncertainty assigned to $\Delta_{c}$ has two contributors i.e. the standard uncertainty assigned to the measurement process on CRM and that assigned to the CRM itself, which is of B type. The $M U$ attributed to $\bar{d}_{C R M}$ is of the same type with that assigned to $\bar{d}_{X}$ therefore it may be expressed as:

$$
u^{2}\left(\bar{d}_{C R M}\right)=\frac{t^{2} S_{C R M}^{2}}{n}+\frac{\delta^{2}}{12}
$$

where $S_{C R M}^{2}$ is the sample variance of the depths given by indentations carried on the CRM, $\delta^{2} / 12$ has the same significance as in Eq.(7), $\mathrm{t}$ is the Student factor.

$S_{C R M}^{2}$ can be calculated using the Eq.(147)

The uncertainty assigned to the CRM is taken from its certificate for $k=1$ and is denoted as $u_{M R C}$. In this instance, the combined uncertainty assigned to $\Delta_{c}$ is:

$$
u_{c}^{2}\left(\Delta_{C}\right)=\frac{t^{2} S_{M R C}^{2}}{n}+\frac{\delta^{2}}{12}+u_{M R C}^{2}
$$

The common practice of the $M U$ assessment is to take into account the uncertainties assigned to machine calibration and that attributed to the operator. But, these contributions are included in the $M U$ assigned to $\bar{d}_{X}$ and to $\Delta_{c}$. Thus, the supplementary addition of the mentioned $M U$ will involve an overestimation of combined uncertainty of the hardness test. 


\subsection{Combined uncertainty calculation}

It is a matter of evidence that the contributions to the overall uncertainty of Rockwell $\mathrm{C}$ hardness test are mutually independent and, consequently, the combined uncertainty $u_{c}$ assigned to $h$ is:

$$
u_{c}\left(h_{R C}\right)=\left[u_{c}^{2}\left(\bar{d}_{X}\right)+u_{c}^{2}\left(\Delta_{c}\right)\right]^{1 / 2}=t^{2}\left(\frac{S_{X}^{2}}{n}+\frac{S_{M R C}^{2}}{n}\right)+\frac{\delta^{2}}{6}+u_{M R C}^{2}
$$

\subsection{Extended uncertainty calculation}

The reference documents such as $[2,17]$ do not specify a calculation methods for extended $M U$ of $h_{R C}$. These reference documents only specify the combined uncertainty of the measurement. ISO 6508 gives clear clues that a Gaussian $p d f$ is assigned to $\bar{d}_{X}$. These are: $t$

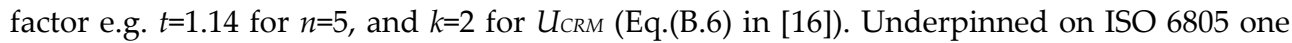
can assign a Gaussian $p d f$ to $\bar{d}_{X}$. Thus, the extended uncertainty can be calculated by multiplying $u_{c}\left(h_{R C}\right)$ with a coverage factor $k$, for instance, $k=2$ for a confidence level of $95 \%$. Thus, the expanded uncertainty assigned to $h_{R C}$ is:

$$
U=U\left(h_{R C}\right)=k\left(u_{c}\left(h_{R C}\right)\right)
$$

According to metallurgical practice, the expanded $M U$ of the test result is:

$$
U(95) 2\left(u_{c}\left(h_{R C}\right)\right)
$$

Thus, the expanded uncertainty of the HRC index is calculated as:

$$
U H R C=U\left(h_{R C}\right) / 0.002
$$

\subsection{Reporting the result}

If the bias of the test is corrected then the result in HRC unit is:

$$
\text { HRC corr }=100-\left(\bar{d}_{X}+\Delta_{c}\right) / 0.002
$$

In this case, the result shall be reported as:

$$
H R C_{\text {corr }} \pm U
$$

on condition that the level of confidence of $U$ or the $k$ value shall be specified.

If the $H R C$ value is not corrected then the $H R C$ index is:

$$
H R C=100-\left(\bar{d}_{X} / 0.002\right)
$$

and the assigned expanded $M U$ to the test result is estimated as:

$$
U+\Delta_{c} / 0.002
$$

In this case, the result shall be reported as: 


$$
\mathrm{HRC} \pm\left(\mathrm{U}+\Delta_{c} / 0.002\right)
$$

\subsection{Numerical example}

\subsubsection{General data about the Rockwell C hardness test}

A Rockwell C hardness test was performed in reproductive conditions on a steel specimen using a Balanta Sibiu Rockwell Machine indirect calibrated on a CRM i.e. a hardness reference block having a certified hardness of $40.1 H R C$ with a $U(95)=0.22 \mathrm{HRC}$. The calibration data obtained on CRM SN 48126 are given in Table 1.

\begin{tabular}{|c|c|c|c|c|c|c|c|c|}
\hline No. & 1 & 2 & 3 & 4 & 5 & Mean & SD & SDmean \\
\hline $\mathrm{d}_{\mathrm{i}}$ & 0,1202 & 0,12 & 0,1192 & 0,1191 & 0,1189 & 0,12 & 0,00058 & 0,00026 \\
\hline $\mathrm{h}_{\mathrm{i}}$ & 0,0798 & 0,08 & 0,0808 & 0,0809 & 0,0811 & 0,08 & 0,00058 & 0,00026 \\
\hline $\mathrm{HRC}_{\mathrm{i}}$ & 39,90 & 40,00 & 40,40 & 40,45 & 40,55 & 40,26 & 0,290 & 0,130 \\
\hline
\end{tabular}

Table 1. The calibration data obtained on CRM SN 48126

Subsequently, according to [16] 5 indentations were carried on the specimen, denoted $X$. The hardness test data for the $X$ specimen are given in Table 2.

\begin{tabular}{|c|c|c|c|c|c|c|c|c|}
\hline No. & 1 & 2 & 3 & 4 & 5 & Mean & SD & SD mean \\
\hline $\mathrm{d}_{\mathrm{i}}$ & 0,1166 & 0,1148 & 0,115 & 0,1154 & 0,117 & 0,12 & 0,00098 & 0,00044 \\
\hline $\mathrm{h}_{\mathrm{i}}$ & 0,0834 & 0,0852 & 0,085 & 0,0846 & 0,083 & 0,08 & 0,00098 & 0,00044 \\
\hline HRC $_{\mathrm{i}}$ & 41,7 & 42,6 & 42,5 & 42,3 & 41,5 & 42,12 & 0,49 & 0,22 \\
\hline
\end{tabular}

Table 2. Test data for the $X$ specimen

From Table 1 and Table 2 it results $\Delta c=-0.15$ HRC.

\subsubsection{MU estimation for the test result according to the classical approach}

The $u_{c}$ assigned to the test result is estimated based on data provided by: testing, calibration procedure, certificate of the CRM and on the specification of operating manual. The data used for $u_{c}$ calculation are given in Table 3 .

\begin{tabular}{|c|c|c|c|c|c|}
\hline No. & Uncertainty source & Symbol & Uncertainty[HRC] & $\begin{array}{c}\text { Evaluation } \\
\text { type }\end{array}$ & Assigned pdf \\
\hline 1 & Indirect calibration & $S_{M-M R C}$ & 0.15 & $\mathrm{~A}$ & Gaussian $^{1}$ \\
\hline 2 & MRC & $U_{M R C}$ & 0.11 & $\mathrm{~B}$ & Triangular $^{1}$ \\
\hline 3 & Sample & $S_{M-X}$ & 0,25 & $\mathrm{~A}$ & Gaussian $^{1}$ \\
\hline 4 & Displaying resolution & $\delta$ & 0.05 & $\mathrm{~B}$ & Triangular $^{1}$ \\
\hline
\end{tabular}

Table 3. The input data used for $u_{c}$ calculation. 1-the assigning of $p d f s$ are taken after [16] but there are no evidences for these assignments.

The combined uncertainty calculated by replacing the values given in Table 3 in Eq.(147) is: 


$$
u_{c}=0.31 \mathrm{HRC}
$$

According to [16] the corrected HRC (see Eq(154)) is:

$$
H R C_{\text {corr }}=41.96 \pm 0.62 \mathrm{HRC}
$$

where $U=0.62 H R C$ is the expanded uncertainty for the $95 \%$ confidence level calculated with a coverage factor $k=2$ according to Anex B of ISO 6805-2.

\subsubsection{MU for the test result according to the multiconvolutional approach}

Whatever is the way of reporting the results, it is quite impossible to assign a confidence level to the expanded uncertainty of the result based on irrefutable arguments. The alternative approach is to consider that the measurand has a uniform $p d f$. The worst case is a uniform $p d f$ having a width equal to the interval of obtained values i.e.

$$
f_{d(x)}=\left\{\begin{array}{c}
\frac{1}{d_{\max }-d_{\min }}, x \in\left[d_{\min }-U_{c c} ; d_{\max }+U_{c c}\right] \\
\text { 0, otherwise }
\end{array}\right.
$$

where $d_{\max }$ and $d_{\min }$ are the maximum and the minimum penetration depths among the five test data. The mean of $f_{d(x)}$ is $\mu=\left(d_{\max }+d_{\min }\right) / 2$ while the half width of the rectangular distribution is $=\left(d_{\max }-d_{\min }\right) / 2$. According to the data given in Table 2 , $\mu=0.1159 \mathrm{~mm}, \Delta_{c}=0.003 \mathrm{~mm}$ and $a=0.0125 \mathrm{~mm}$. As shown in \&3.2.3 the probability that the mean $m$ of a 5 reproductive outcomes, each one being uniformly distributed in $[-a, a]$, to belong to the interval $[m-a / 10 ; m+a / 10]$ is $29.4 \%$. while in $[m-a / 5 ; m+a / 5]$ is $57.64 \%$ Thus, the conventional true value of the penetration depth lies in the interval [0.1157; 0.1161$] \mathrm{mm}$ with a confidence level of 55\%. In this instance, the HRC index of the specimen could be reported as:

$$
H R C \text { corr }=41.89 \pm 0.55 H R C
$$

for the $55 \%$ confidence level calculated on the basis of $p d f$ of mean.

The confidence level for $\left|\frac{m-\mu}{a}\right| \leq \frac{2}{5}$ is about $99 \%$. In this case, Rockwell C index could be reported as:

$$
H R C \text { corr }=41.89 \pm 1.1 H R C
$$

The above result should be interpreted as the HRC conventional true value of the specimen lies in the interval $[40.79,42.99]$ (HRC) with 99\% confidence level. For the conventional case adopted by ISO 6805 and presented above, the $99 \%$ confidence level corresponds to a coverage factor $k=3$ i.e. to a $U(99 \%)=0.93 H R C$. By comparison, the reported test result, according to [16], as $H R C_{\text {corr }}=41.96 \pm 0.93 H R C$ with $99 \%$ confidence level or, according multiconvolutional approach, as $H R C_{\text {corr }}=41.89 \pm 1.10 \mathrm{HRC}$ seems to be quite the same to some extent depending on the rigor claimed by the client. But, from scientific and mathematical statistics point of view one may feel comfortable to use a founded test result having assigned a little bit larger uncertainty than to use a doubtful one. 


\subsection{Discussion}

The most important finding of this case study is that, when dealing with a measurand whose assigned $p d f$ is not known or is insufficiently documented, the best approach is to consider it has a uniform distribution. The interval of variance of the outcomes may be considered, at first glance, as the distribution width. Another important issue is that for estimating $M U$ using the multiconvolutional approach, only data provided by the testing process are used, while the classical approach uses supplementary data. A sound question regarding the multiconvolutional approach is how to decrease the $M U$ i.e. to improve the test result quality? The classical solution is that the experimentalist should increase the number of reproductive or repetitive tests. The common practice of five tests appears insufficient, but ten tests should be acceptable. Using a ten-fold convolved uniform $p d f s$ one can describe quite accurately the probability of mean displacement about the conventional true value $(\mu)$ with at least $0.1 \mu$ increment.

\section{Summaries}

This article addresses a more meaningful approach for measurement uncertainty estimation, particularly in the metallurgical test field. The chapter contributes to the state of the art by the development of a consistent approach for calculating the pdfs of the sample mean statistic, of the variance and of $t$ parameter. To this end, the concepts of probabilistic theory and the derivation of the main $p d f s$ used for measurement uncertainty evaluation as Poisson, Gauss are presented briefly. The theoretical backgrounds are presented in the paper in the aim to make clear for an experimentalist the specific $p d f s$ to be assigned to a measurand. A considerable part of the chapter addresses the deriving of the pdfs of the compound variables as $Y=a X+b, Y=\left(X_{1}+\ldots+X_{n}\right) / n, Y=X^{2}, Y=\sqrt{ } X, Y=X_{1} / X_{2}$ because these form the basis for deriving the probability density functions of sample mean, of variance, of Student and of Fisher-Sedecor. The pdfs of sample mean and of Chi distribution are derived in extenso following the convolution approach because it provides an easy-to-use and intuitive way to understand how these distributions should be applied for measurement uncertainty estimation. Thus, this approach allows an in-depth understanding of the mathematical formulas in order to avoid their usage exclusively based upon the mathematical literature without understanding of or without concern about the appropriateness to the case addressed. An important contribution of the chapter is the argument for using a number of repeated test, $n$, as the number of degrees of freedom and not $n-1$ as is common practice. The last part of the chapter deals with measurement uncertainty estimation using GUM method because it is required by EN ISO/CEI 17025. The GUM approach was applied for the uncertainty estimation of the Rockwell C hardness test according to the ISO 6825 standard. As is underlined in the chapter, this standard does not provide clear evidence for assigning Gaussian distribution to the hardness HRC. Alternative approach to estimate the uncertainty of the Rockwell C hardness test result is given based on the $p d f$ of the sample mean obtained by 5 -fold convolved product of the uniform distribution assigned to the measurand. 
The entire chapter is designed to emphasize the risk of wrongly estimating the test result uncertainty due to erroneous assumptions regarding the $p d f$ attributed to the measurand or to the influence factors of the uncertainty budget.

\section{Author details}

Ion Pencea

Metallic Material's Science and Engineering Department, Materials Science and Engineering Faculty, University "Politehnica" of Bucharest, Bucharest, Romania

\section{References}

[1] EN ISO/IEC 17025:2005, General requirements for the competence of testing and calibration laboratories

[2] Guide to the Expression of Uncertainty in Measurement, Edition, ISO, Geneva, 1995,

[3] Sfat, C.E, (2011) Quality Assurance of Test Results, in: Saban, R., editor, Treatise of Science and Engineering of Metallic Materials, AGIR Publisher, Bucharest, Romania, pp 1272-1287.

[4] Eurolab Technical Report 1/2007-Measurement Uncertainty Revised, pp 47-50

[5] Cuculescu, I., (1998) Probability Theory, All Publisher, Bucharest, Romania, 510p.

[6] Ventsel, H., (1973) Theorie des Probabilities, MIR Publisher, Moscou, 565p

[7] Dehoff, R.T., Rhines, F.N., (1968) Quantitative Microscopy, McGrow-Hill Book Company, 421p.

[8] ISO 3534-1:2006, Statistics -Vocabulary and symbols - Part 1: General statistical terms and terms used in probability

[9] Renyi, A., (1970) Probability Theory. North-Holland, Amsterdam, 458p.

[10] Frank Killmann, F., von Collani, E., (2001) A Note on the Convolution of the Uniform and Related Distributions and Their Use in Quality Control, Heldermann Verlag Economic Quality Control, Vol 16, No. 1, pp.17 - 41

[11] ISO 5725 (6 parts), Accuracy (trueness and precision) of measurement methods and results

[12] Pencea, I., (2011) Methods and Techniques of Instrumental Analysis of Materials in: Saban, R., editor, Treatise of Science and Engineering of Metallic Materials, AGIR Publisher, Bucharest, Romania, pp 1057-1234.

[13] VIM-International Vocabulary of Metrology - Basic and General Concepts and Associated Terms, JCGM 200:2012,

[14] EA Guideline EA-4/16: Expression of Uncertainty in Quantitative Testing EA 2003, Guidelines for the estimation of uncertainty in environmental measurement, (www.european-accreditation.org)

[15] Popescu, N., Bunea, D., Saban, R., Pencea, I., (1999) Material Science for Mechanical Engineering, v1, Fair Partner Publisher, Bucharest, Romania, 270p.

[16] ISO 6508-1:2005, Metallic materials -- Rockwell hardness test -- Part 1: Test method (scales A, B, C, D, E, F, G, H, K, N, T)

[17] EA Guideline EA-10/16: EA Guidelines on the Estimation of Uncertainty in Hardness Measurement, (www.european-accreditation.org), 